ARTICLE

\title{
Engineering immunomodulatory and osteoinductive implant surfaces via mussel adhesion-mediated ion coordination and molecular clicking
}

Tao Wang 1,2,3,5, Jiaxiang Bai (10 ${ }^{4,5}$, Min Lu', Chenglong Huang ${ }^{3}$, Dechun Geng ${ }^{4}$, Gang Chen $^{3}$, Lei Wang ${ }^{1}$, Jin Qi ${ }^{1 凶}$, Wenguo Cui ${ }^{1 凶} \&$ Lianfu Deng ${ }^{1 凶}$

Immune response and new tissue formation are important aspects of tissue repair. However, only a single aspect is generally considered in previous biomedical interventions, and the synergistic effect is unclear. Here, a dual-effect coating with immobilized immunomodulatory metal ions (e.g., $\mathrm{Zn}^{2+}$ ) and osteoinductive growth factors (e.g., BMP-2 peptide) is designed via mussel adhesion-mediated ion coordination and molecular clicking strategy. Compared to the bare $\mathrm{TiO}_{2}$ group, $\mathrm{Zn}^{2+}$ can increase $\mathrm{M} 2$ macrophage recruitment by up to $92.5 \%$ in vivo and upregulate the expression of $\mathrm{M} 2$ cytokine IL-10 by $84.5 \%$; while the dual-effect of $\mathrm{Zn}^{2+}$ and BMP-2 peptide can increase M2 macrophages recruitment by up to $124.7 \%$ in vivo and upregulate the expression of M2 cytokine IL-10 by $171 \%$. These benefits eventually significantly enhance bone-implant mechanical fixation $(203.3 \mathrm{~N})$ and new bone ingrowth (82.1\%) compared to the bare $\mathrm{TiO}_{2}(98.6 \mathrm{~N}$ and $45.1 \%$, respectively). Taken together, the dual-effect coating can be utilized to synergistically modulate the osteoimmune microenvironment at the bone-implant interface, enhancing bone regeneration for successful implantation.

\footnotetext{
${ }^{1}$ Department of Orthopaedics, Shanghai Key Laboratory for Prevention and Treatment of Bone and Joint Diseases, Shanghai Institute of Traumatology and Orthopaedics, Ruijin Hospital, Shanghai Jiao Tong University School of Medicine, 197 Ruijin 2nd Road, 200025 Shanghai, P. R. China. ${ }^{2}$ Department of Orthopaedics, Shanghai General Hospital, Shanghai Jiao Tong University School of Medicine, 85 Wujin Road, 200080 Shanghai, P. R. China. ${ }^{3}$ Jiaxing Key Laboratory of Basic Research and Clinical Translation on Orthopedic Biomaterials, Department of Orthopaedics, The second Affiliated Hospital of Jiaxing University, 1518 North Huancheng Road, 314000 Jiaxing, P. R. China. ${ }^{4}$ Department of Orthopaedics, The First Affiliated Hospital of Soochow University, 188 Shizi Street, Suzhou 215006 Jiangsu, P. R. China. ${ }^{5}$ These authors contributed equally: Tao Wang, Jiaxiang Bai. 凶email: jinjin838@hotmail.com; wgcui80@hotmail.com; If_deng@126.com
} 
B one implantation using nonliving materials (e.g., metals, polymers, and ceramics) as bone substitutes has proven to be an efficient clinical method for bone fracture fixation, joint arthroplasty, spinal reconstruction, and so on ${ }^{1-3}$. Clinically, the success rate of integration between implants and bone tissues exceeds $95 \% 4,5$, while the failure rate of early implantation was about $1.2 \%$ due to the development of fibrous tissue between the implants and the surrounding bone tissues in the healing period ${ }^{6}$. The general problem in these exogenous biomaterials is their bioinertness, lacking in bioactivities to completely adapt to the complex physiological bone regeneration process. Tissue regeneration involves three indispensable stages: (i) immune action, (ii) cell proliferation and new tissue formation, and (iii) remodeling and maturation ${ }^{7,8}$. The bio-inertness of exogenous biomaterials thus is inclined to lead to fibrotic encapsulation, implant loosening, or implantation failure. Traditional studies regarding bone implants predominantly focused on optimizing the osteogenic capacity, with some inert bone implants designed to evade immune response and others introduced with various bioactive moieties (e.g., peptides, growth factors, protein, and even ions) for promoting osteogenesis in vitro and bone-to-implant osseointegration $^{9-11}$. However, these implants may not completely adapt to the in vivo microenvironment, thus leading to some inconsistent results in vivo ${ }^{12}$ majorly due to the uncontrolled local immune responses triggered by exogenous biomaterials. Thus in order to develop implants with efficient bone regeneration, it is inadequate to merely emphasize the direct osteogenesis while ignoring the local immune microenvironment. To design an ideal bone implant, the synergy of direct osteogenicity and immunomodulatory function should be considered to precisely match the mechanisms of bone regeneration process and achieve satisfactory osteogenesis at the bone-to-implant interfaces.

Recent studies on osteoimmunology further revealed that immune microenvironments also play an important role in bone tissue formation ${ }^{7,13,14}$. In different microenvironments, macrophages polarize into classically activated macrophages (M1) or alternatively activated macrophages (M2). The pro-inflammatory M1 macrophages activate inflammation and promote fibrosis while the pro-healing M2 macrophages coordinate tissue healing processes by activating stem/progenitor cells and remodeling extracellular matrix for regeneration ${ }^{15,16}$. As is found in previous studies, an efficient and timely switch from M1 to M2 macrophage phenotype was essential for bone healing and osteointegration around bone implants, creating a favorable osteoimmune environment via the increased production of anti-inflammatory (e.g., IL-10) and pro-osteogenic (e.g., BMP-2 and VEGF) cytokines ${ }^{17}$. To this end, various efforts on surface bioengineering of the bone implants, such as optimizing surface physical properties $^{18-21}$ (e.g., topography, wettability, charge, etc.) and introducing cytokines ${ }^{22-24}$ (e.g., interleukin-4 (IL-4), lipoxin A4 (LXA4), etc.) or active metal ions ${ }^{25-28}$ (e.g., $\mathrm{Ca}^{2+}, \mathrm{Zn}^{2+}, \mathrm{Sr}^{2+}$, etc.), have been made to regulate the local immune microenvironment and ameliorate the final outcomes of bone regeneration and osseointegration around bone implants in vivo.

Surface bioengineering possesses remarkable superiority in introducing osteoinductive and immunomodulatory activities onto a bone implant because it allows surface modification with various bioactivities for diversified requirements ${ }^{29-32}$. Multifunctional bone implants capable of co-regulating stem/progenitor cells and immune cells could conceivably be readily obtained by surface co-modification of relevant biological signals, which may construct a benign microenvironment suitable for complete bone regeneration. To date, various physical or chemical means have been vigorously developed for surface bioengineering. For instance, layer-by-layer assembly, Langmuir-
Blodgett deposition silanization, anodization, acid etching, and ion doping were widely used to add implant surfaces with different bioactive moieties (e.g., peptides, proteins, and even ions) ${ }^{33-36}$. Since physical methods have persistent drawbacks of serious molecular leakage and the lack of long-term activity, current surface bioengineering strategies for bone implants mainly rely on chemical conjugations ${ }^{37,38}$. Traditional chemical methods, however, mostly involve tedious chemical reactions as well as complicated surface treatment technologies ${ }^{1,39}$. Apart from potential damage to the bioactive molecules, the complex procedures of these methods also hinder their application in multi-modification due to the low controllability and poor operability. Therefore, simple and biocompatible surface approaches capable of efficiently conjugating multiple bioactivities are highly desired particularly for the design of dualfunctional bone implants with both osteoinductive and immunomodulatory functions to match the mechanism of bone regeneration.

Biomimetic strategies have emerged as a promising approach for the multi-modification. In 2007, Lee et al. developed a novel surface chemistry via dopamine polymerization ${ }^{40}$. The method was inspired by the molecular adhesion mechanism of marine mussel foot proteins (Mfps), in which repetitive catecholic amino acids (3,4-dihydroxy-L-phenylalanine, DOPA) contribute to strong surface adhesion ${ }^{41}$. Likewise, catechol-rich poly(dopamine) can also achieve robust molecular adhesion to virtually all kinds of substrates ${ }^{42}$. In addition, the catechol residues on the surface enable not only simple conjugations of amino- or thiol-containing biomolecules via Michael addition or Schiff base reaction but also spontaneous coordination with bioactive metal ions ${ }^{43-45}$. These advantages indicate the mussel-inspired surface strategy has the potential to co-modify bone implants with osteoinductive biomolecules and immunomodulatory active ions. However, the critical problem of this strategy is the random consumption of active groups (e.g., amino and thiol), which would interfere with the functions of conjugated biomolecules ${ }^{38,46}$. Recently, Pan et al. ${ }^{1}$ designed two musselderived biomimetic peptides for simple biomodification of $\mathrm{Ti}$ implants through robust catechol/titanium dioxide $\left(\mathrm{TiO}_{2}\right)$ coordinative interactions. The highly biomimetic peptides capped with RGD- or OGP-derived sequences could improve not only the biocompatibility of $\mathrm{Ti}$ implants but also the efficiency of osteogenicity, osseointegration, and mechanical stability in vivo. The strategy provides a clear chemical binding on implants surfaces yet the uncontrolled biomolecular conjugation, particularly for multi-modification, which is not conductive to the reproducibility of a multi-bioactive surface. Given this, we designed an improved biomimetic strategy by combining mussel-like adhesion with bioorthogonal click reaction, a specific and biocompatible chemistry ${ }^{47-49}$. We hypothesize this strategy which involves a bioclickable way for biomolecular conjugation and a coordination means for ion loading would provide a promising solution for surface engineering of osteoinductive and immunomodulatory bone implants.

In this work, we chose medical titanium (Ti) screw as implant model since Ti materials are widely used in orthopedic and dental surgery. To integrate the mussel adhesion mechanism with bioorthogonal click chemistry, we first synthesize a DOPAcontaining peptide with bioclickable group dibenzylcyclooctyne (DBCO) $)^{50}$ (Fig. 1A). Next, the clickable mussel-derived peptide is stably bound onto $\mathrm{Ti}$ screws via metal-catechol coordination. Then, an immunoactive metal zinc ion $\left(\mathrm{Zn}^{2+}\right)$ capable of polarizing macrophages to the anti-inflammatory M2 phenotype is coordinated with the catechol residues to generate an immunoactive surface. For direct osteogenicity, a BMP-2-derived synthetic peptide capped with azido group is synthesized and 

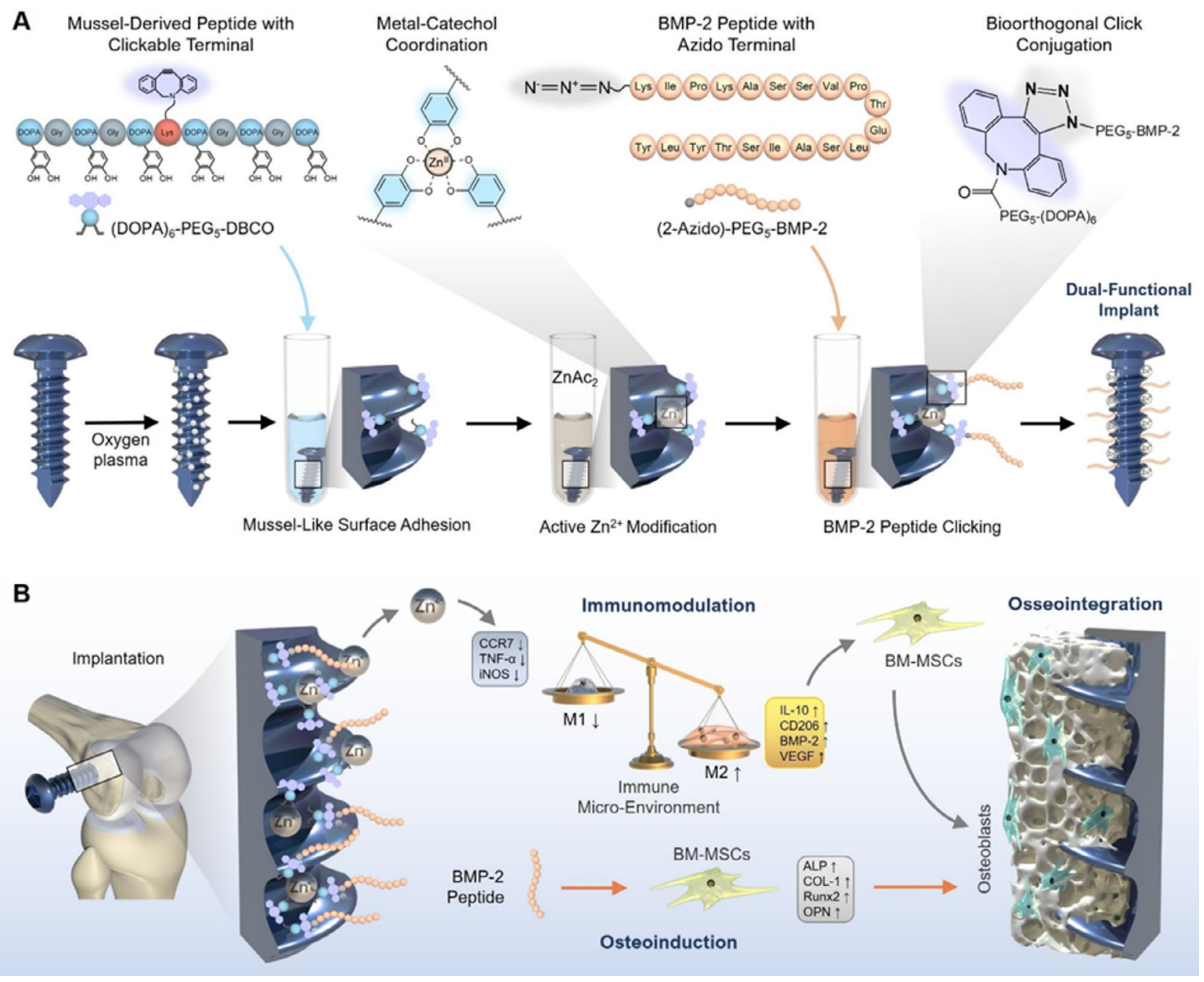

Fig. 1 Design strategy for engineering immunomodulatory and osteoinductive implant surfaces. A Schematic illustration of the mussel-derived peptide for ion coordination and biomolecular click conjugation on a medical Ti screw. B In a bone implant model, the $\mathrm{Zn}^{2+}$ and BMP-2 peptide co-modified Ti screw shows osteoinductive and immunomodulatory dual functions in vivo, synergistically enhancing the interfacial osteogenesis and the intra-bone implant integration after implantation.

conjugated with surface DBCO groups via a bioorthogonal cycloaddition chemistry. After successful synthesizing the implants, the efficiency of mussel adhesion-mediated ion coordination and molecular clicking between $\mathrm{Zn}^{2+}$ modification and BMP-2 peptide conjugation is evaluated, respectively. Then, the synergetic effect of immunoactive $\mathrm{Zn}^{2+}$ and osteoinductive BMP2 peptide on macrophage polarization and interfacial osteogenesis in vitro and in vivo are investigated and discussed. Overall, this study elucidates the synergy of direct osteogenicity and immunomodulatory function on osteogenesis. It may also provide a simple and efficient solution for the engineering of osteoinductive and immunomodulatory implants to precisely adapt to the favorable microenvironment in vivo for bone regeneration.

\section{Results and discussion}

Mussel-derived peptide synthesis and surface modification. The mussel-derived peptide with clickable DBCO group was synthesized by solid-phase peptide synthesis strategy with a Mfps-like peptide mimicking method ${ }^{39,47}$. A commercially available acetonide-protected Fmoc-DOPA (acetone)-OH was used to introduce catecholic amino acid DOPA into the peptide sequence. To preserve enough catechol groups for $\mathrm{Zn}^{2+}$ coordination and accessible DBCO groups for biomolecular clicking after peptide adhesion on Ti surfaces, hexavalent DOPA units with one amino acid interval and DBCO with a long polyethylene glycol (PEG) chain were used to prepare the mussel-derived peptide Ac(DOPA)-G-(DOPA)-G-(DOPA)-K[(PEG $)-(\mathrm{Mpa})-(\mathrm{Mal}-$

DBCO)]-(DOPA)-G-(DOPA)-G-(DOPA) ((DOPA) ${ }_{6}-\mathrm{PEG}_{5}$ DBCO) (Fig. 2A and Supplementary Fig. 1). In addition, a peptide (KIPKASSVPTELSAISTLYL) derived from the 73 to 92 amino acid fragment of the BMP-2 finger epitope was conjugated with two-azidoacetic acid and $\mathrm{PEG}_{5}$-carboxyl coupling, respectively (Fig. 2B and Supplementary Fig. 1). The obtained azido-capped BMP-2-derived peptide (Azido-KIPKASSVPTELSAISTLYL, (2Azido)-PEG - -BMP-2) thus could be easily connected with (DOPA $)_{6}-\mathrm{PEG}_{5}-\mathrm{DBCO}$-bound surfaces, providing a flexible surface modification strategy. The two synthesized peptides were first purified by high-performance liquid chromatography (HPLC, purity > 95\%) (Supplementary Fig. 2). Electrospray ionization mass spectrometry (ESI-MS) was further used to confirm their molecular structures. The monoisotopic mass $[\mathrm{M}+2 \mathrm{H}]^{2+}$ of $(\mathrm{DOPA})_{6}-\mathrm{PEG}_{5}-\mathrm{DBCO}$ and $[\mathrm{M}+2 \mathrm{H}]^{2+}$ of $(2-$ Azido)-PEG - BMP-2 were found at $1036.69 \mathrm{Da}$ and $1247.63 \mathrm{Da}$, which corresponded to the theoretical molecular weight at 2070.18 Da and 2492.86 Da, respectively (Fig. 2C, D). It is worth 

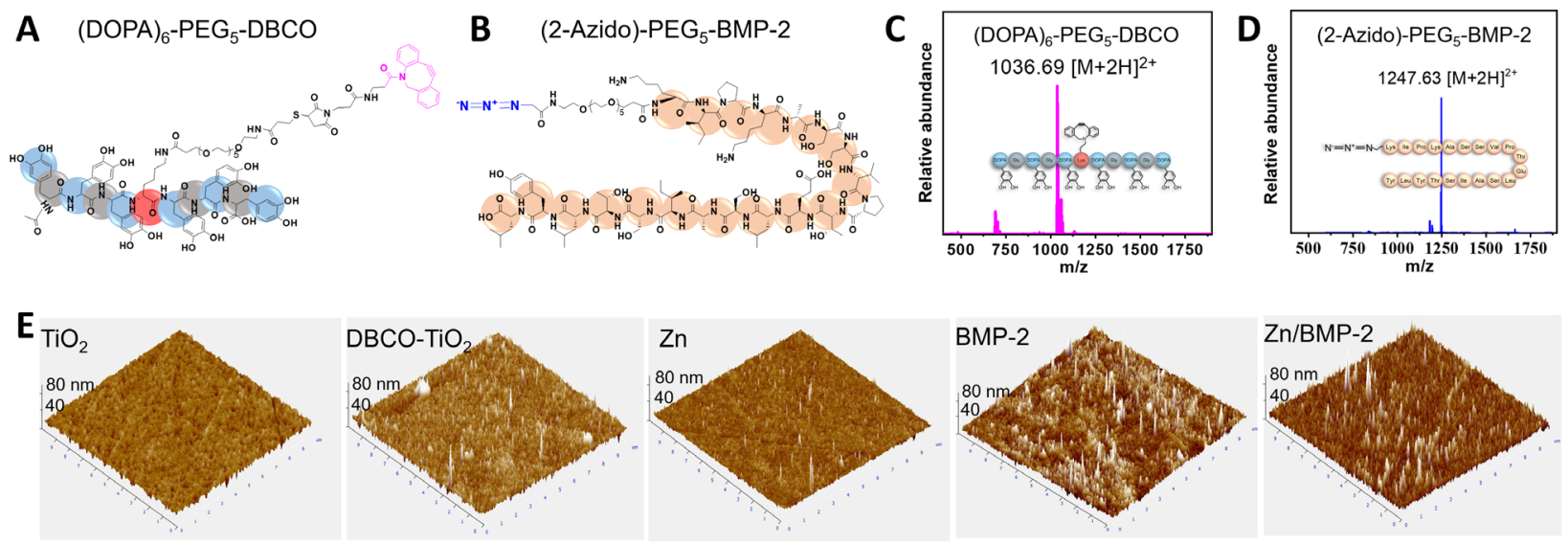

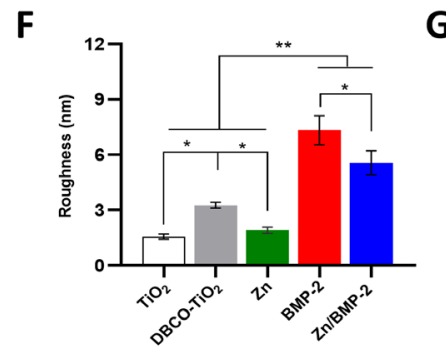

G
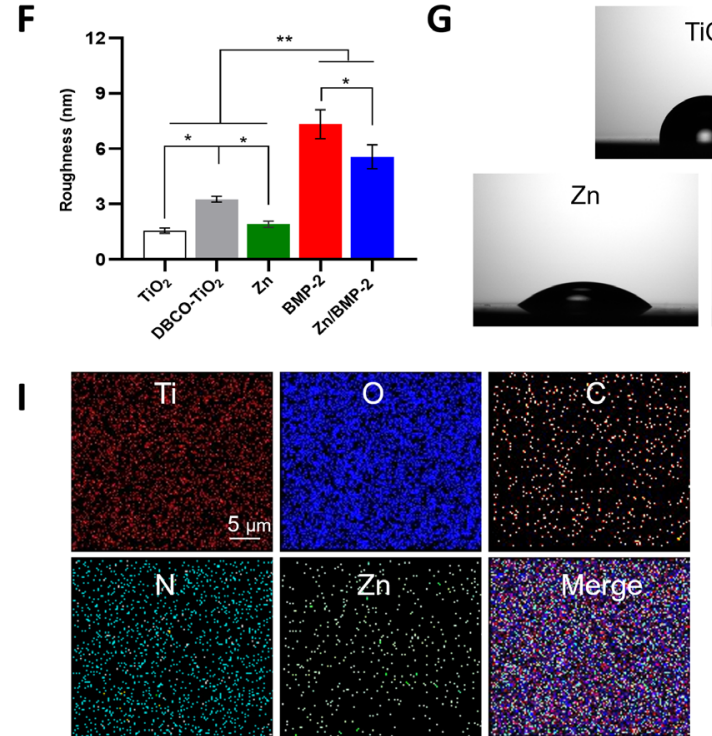

$\mathbf{L}$

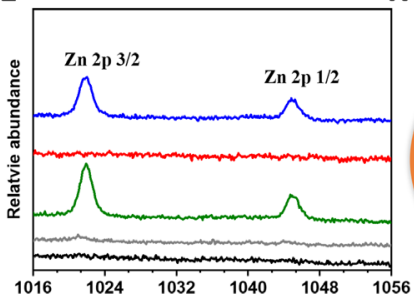

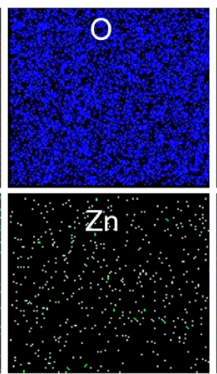

M
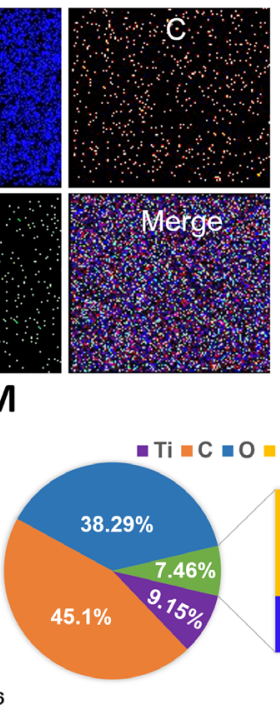

$\mathrm{TiO}_{2}$

DBCO- $\mathrm{TiO}_{2}$

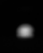

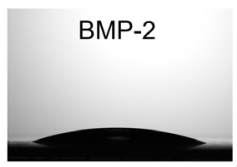

Zn/BMP-2

J

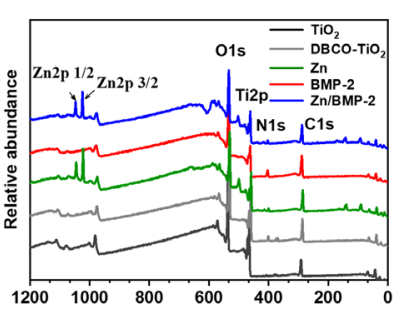

K

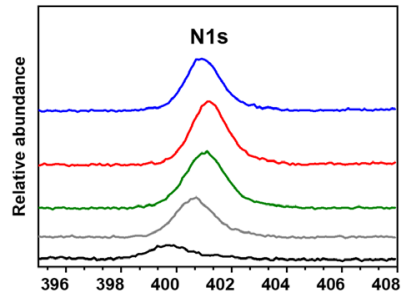

H

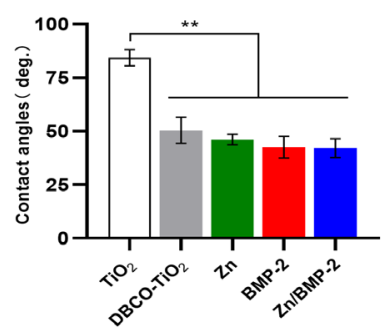

Fig. 2 Characterizations of clickable peptide and $\mathbf{Z n}^{2}+$ and BMP-2 peptide co-modified surface. A, B The molecular structures of (DOPA) ${ }_{6}-P E G_{5}-D B C O$ and (2-Azido)-PEG 5 -BMP-2. C, D ESI-MS spectra of the two synthetic peptides. E AFM images of different surfaces and (F) quantitation of the suface roughess with the different modified surfaces ( $n=3$ independent samples per group, by a one-way ANOVA with a Tukey's post hoc test for multiple comparisons). Data are reported as mean $\pm \mathrm{SD},{ }^{\star} p<0.05,{ }^{\star \star} p<0.01$ ). G The water contact angles of different surfaces and (H) the quantitative results ( $n=3$ independent samples per group, by a one-way ANOVA with a Tukey's post hoc test for multiple comparisons. Data are reported as mean \pm SD, $\left.{ }^{\star \star} p<0.01\right)$. I SEM-EDS elemental mapping for the $\mathrm{Zn}^{2+}$ and BMP-2 peptide co-modified surface ( $\left.\mathrm{Zn} / \mathrm{BMP}-2\right)(\mathrm{scar}$ bar $=5 \mu \mathrm{m}$, three independent experiments). J-L XPS analysis of the bare and modified $\mathrm{TiO}_{2}$ surface (DBCO-TiO $2, \mathrm{Zn}, \mathrm{BMP}-2$ and $\mathrm{Zn} / \mathrm{BMP}-2$. M Quantitative elemental analysis according to XPS. N Changes of N 1 s signal in the XPS spectrum of the Zn/BMP-2 surface after incubated in DMEM for 2 weeks. $\mathbf{O} Z \mathrm{n}^{2+}$ release profiles of the $\mathrm{Zn} / \mathrm{BMP}^{2}$ surface in PBS solution; red (left) and blue (right) represent the non-accumulative and accumulative $\mathrm{Zn}{ }^{2+}$ release, respectively. Exact $P$ values were given in the Source Data file.

mentioning that the azido-capped osteogenic peptide (2-Azido)$\mathrm{PEG}_{5}-\mathrm{BMP} 2$ still showed excellent osteogenic activity (Supplementary Fig. 3). Incubating bone marrow mesenchymal stem cells (BM-MSCs) with (two-Azido)- PEG $_{5}$-BMP-2 peptide could elicit potent alkaline phosphates (ALP) activity and matrix mineralization, demonstrating the efficient osteoinductive ability of this molecularly modified BMP-2 peptide. These results indicated the successful preparation of the clickable mussel-derived peptide and azido-capped osteogenic biomolecule.

With the two peptides, surface coating on Ti-based substrates was then performed to prepare a $\mathrm{Zn}^{2+}$ and BMP-2 peptide comodified surface. $\mathrm{TiO}_{2}$-deposited quartz substrate (noted as $\mathrm{TiO}_{2}$ ), the control group in the following experiments, was used to mimic the surface of medical $\mathrm{Ti}$ biomaterials in all in vitro 
investigations. (DOPA) $)_{6}-\mathrm{PEG}_{5}-\mathrm{DBCO}$-coated substrates (noted as $\mathrm{DBCO}-\mathrm{TiO}_{2}$ ) were prepared by incubating the $\mathrm{TiO}_{2}$-deposited quartz in phosphate buffer saline solution (PBS, $0.02 \mathrm{mM}$, $\mathrm{pH}=7.2$ ) with the mussel-derived peptide. The catechol residues on peptide-coated substrates were then coordinated with $\mathrm{Zn}^{2+}$ after immersion into zinc acetate $\left(\mathrm{ZnAc}_{2}\right)$ solution to obtain $\mathrm{Zn}^{2+}$-loaded surface (noted as $\mathrm{Zn}$ ). Finally, (2-Azido)- $\mathrm{PEG}_{5}{ }^{-}$ BMP-2 was conjugated through bioorthogonal click chemistry to prepare a $\mathrm{Zn}^{2+}$ and BMP-2 peptide co-modified surface (noted as $\mathrm{Zn} / \mathrm{BMP}-2$ ). A BMP-2-modified surface without loading $\mathrm{Zn}^{2+}$ (noted as BMP-2) was also prepared as a control. Quartz-crystal microbalance $(\mathrm{QCM})$ was used to monitor the peptide grafting densities of (DOPA) ${ }_{6}-\mathrm{PEG}_{5}-\mathrm{DBCO}$ and (2-Azido)- $\mathrm{PEG}_{5}-\mathrm{BMP}^{-}$ $2^{51}$. As shown in Supplementary Fig. $4 \mathrm{~A}$, (DOPA) $)_{6}-\mathrm{PEG}_{5}-\mathrm{DBCO}$ could be steady bound onto the QCM chips and the maximal grafting density was about $489 \mathrm{ng} / \mathrm{cm}^{2}$, indicating the high efficiency and spontaneous adhesion onto $\mathrm{TiO}_{2}$-deposited quartz substrate surface. The grafting density in our study was higher than that of Pan's work $\left(363 \mathrm{ng} / \mathrm{cm}^{2}\right)^{1}$, mainly due to the improved binding affinity of mussel-adhesion peptide resulting from the increased number of catechol groups. Then, the DBCOmodified $\mathrm{TiO}_{2}$ substrates were incubated with azido-capped BMP-2-derived peptides for bioorthogonal conjugation (Supplementary Fig. 4B). The click reaction started in a few minutes, and the maximal grafting density for (2-Azido)-PEG - -BMP-2 was $140 \mathrm{ng} / \mathrm{cm}^{2}$, which was comparable to the results in previous reports on the immobilization of BMP-2 on chitosan-grafted Ti surfaces $\left(50 \mathrm{ng} / \mathrm{cm}^{2}\right)^{52}$ and the polydopamine-coated nanofibers $\left(124 \mathrm{ng} / \mathrm{cm}^{2}\right)^{53}$, respectively. The changes of surface roughness after peptide modification were first checked by atom force microscope (AFM) (Fig. 2E). A clear change was observed after $(\mathrm{DOPA})_{6}-\mathrm{PEG}_{5}-\mathrm{DBCO}$ peptide modification. Although the $\mathrm{Zn}^{2+}$-modification led to a negligible change, the second peptide modification with (2-Azido)- PEG $_{5}$-BMP-2 resulted in a significant increase in surface roughness. Quantitative analysis confirmed the results (Fig. 2F), indicating the efficiency of peptide modification through mussel-like adhesion and subsequent peptide clicking. Likewise, the surface wettability was significantly improved after $\mathrm{Zn}^{2+}$ or BMP-2 peptide modification (Fig. $2 \mathrm{G}, \mathrm{H}$ ), probably due to the hydrophilicity of surface chelated $\mathrm{Zn}^{2+}$ and the amino acid sequence of BMP-2 peptide. The successful $\mathrm{Zn}^{2+}$-modification was confirmed by energydispersive X-ray spectrometry (EDS) elemental mapping and $\mathrm{X}$-ray photoelectron spectroscopy (XPS). Although scanning electron microscopy (SEM) analysis showed no significant differences on different surfaces after co-modification (Supplementary Fig. 5), EDS elemental mapping revealed homogeneously distributed zinc on the surface after treatment with $\mathrm{Zn}^{2+}$ (Fig. 2I). Surface elemental compositions were further determined by XPS to further confirm $\mathrm{Zn}^{2+}$ and BMP-2 peptide co-modification (Fig. 2J-L). On the $\mathrm{TiO}_{2}$-deposited surface ( $\mathrm{TiO}_{2}$ group), only the signal peaks of carbon, Ti and oxygen were found, while in the groups of $\mathrm{Zn}$ and $\mathrm{Zn} / \mathrm{BMP}-2$, Zn 2p3/2 and $\mathrm{Zn} 2 p 1 / 2$ signal peaks at $1021.75 \mathrm{Da}$ and $1044.85 \mathrm{Da}$ were found (Fig. 2L). In addition, the $1 \mathrm{~s}$ signal of nitrogen ( $\mathrm{N} 1 \mathrm{~s}$ ) was found on the DBCO-TiO ${ }_{2}$, BMP-2 and $\mathrm{Zn} / \mathrm{BMP}-2$ groups $(400.13 \mathrm{eV})$. A gradual increase in $\mathrm{N} 1 s$ was also observed when the $\mathrm{DBCO}-\mathrm{TiO}_{2}$ surface was further modified with (2-Azido)-PEG - -BMP-2 (i.e., the BMP-2 and $\mathrm{Zn} /$ BMP-2 groups) (Fig. 2K). To be exact, the N/Ti atomic ratio increased from 0.052 (the $\mathrm{TiO}_{2}$ group) to 0.686 (the BMP-2 group); the $\mathrm{Zn} / \mathrm{Ti}$ atomic ratio increased from 0.000 (the $\mathrm{TiO}_{2}$ group) to 0.003 (the BMP-2 group) (Supplementary Table 1). Quantitative analysis revealed that atom percentages of zinc and nitrogen on the $\mathrm{Zn} / \mathrm{BMP}-2$ surface were $2.57 \%$ and $4.89 \%$, respectively, indicating the efficiency of $\mathrm{Zn}^{2+}$ ion and BMP-2 peptide co-modification (Fig. 2M). Then, the durability of BMP-2 on the surface was evaluated by incubating the Zn/BMP2 substrate in Dulbecco's modified Eagle's medium/F12 (DMEM/F12, $37^{\circ} \mathrm{C}$ ) for 2 weeks. As shown in Fig. $2 \mathrm{~N}$, the intensity of N $1 s$ signal in XPS showed a slight decrease of $<15 \%$. In addition, the durability of the coated clickable peptide labelled by a FITC probe was further checked to confirm the bioactivity. Despite of incubation in DMEM/F12 for 2 weeks, the intensity of fluorescence on the clickable peptide-modified $\mathrm{TiO}_{2}$ surface $(\mathrm{Zn} /$ BMP-2 group) did not show significant reduction (Supplementary Fig. 6). Thus, it could be concluded that immobilized BMP-2 peptide is highly stable, probably due to the covalent bonding between DBCO group and azido group via bioorthogonal click chemistry. In addition, the release of $\mathrm{Zn}^{2+}$ from the $\mathrm{Zn} / \mathrm{BMP}-2$ co-modified surface to PBS was determined by an inductively coupled plasma-atomic emission spectrometry (ICP-AES, JY2000-2, France). The $\mathrm{Zn}^{2+}$ release was also comparable to previous reported $\mathrm{Zn}^{2+}$-modified surface by sequential sulfonation and magnetron sputtering ${ }^{28}$. Specifically, a burst $\mathrm{Zn}^{2+}$ release was observed on the first day $(0.145 \mathrm{ppm})$, and the release slowed down in the following days and reached a steady state (0.04 ppm) lasting 3-4 weeks. Furthermore, the zinc release from FBS-free DMEM and 10\% FBS-coanting DMEM were investigated. During the 4-week observation, all the cumulative profiles (Supplementary Fig. 7) showed similar release characteristics. Therefore, the $\mathrm{Zn}^{2+}$ concentration in the local microenvironment around the $\mathrm{Zn} / \mathrm{BMP}-2$ co-modified Ti implant in vivo is probably at a similar level as in vitro. In a word, these results collectively indicated that the $\mathrm{TiO}_{2}$ based surfaces were successfully comodified with Zn/BMP-2 and had potential to show long-term bioactivity.

Surface cytocompatibility in vitro. After passage 3 , the cells were detached with trypsin, and then the cell surface markers were determined by flow cytometry (LSRFortessaTM X-20, BD, USA) analysis to identify the purity of BM-MSCs. As shown in Supplementary Fig. 8, high expressions of CD29 (99.8\%) and CD90 (98.9\%) and extremely low expressions of the hematopoietic marker CD45 (1.7\%) and CD34 (3.1\%) were detected, indicating the high purity of BM-MSCs as well as the feasibility of employing these cells in following studies.

The in vitro biocompatibility of $\mathrm{Zn}^{2+}$ or BMP-2 modified surfaces was investigated by seeding leukemia cells in mouse macrophage (RAW 264.7 cells) and BM-MSCs on the surfaces. The Live/Dead staining was first carried out and a slight reduction in the number of dead cells was observed on the $\mathrm{Zn}^{2+}$, BMP-2 peptide, or their co-modified surfaces compared to the bare $\mathrm{TiO}_{2}$ surface (Fig. 3A). To further quantify the cell viability, RAW264.7 and BM-MSCs were stained by Annexin V/ Propidium iodide (PI) staining and analyzed with the flow cytometry (LSRFortessaTM X-20, BD, USA). These results showed the mean living cell (Annexin V-, PI-) percentage on Zn/BMP-2 co-modified surface was up to 94\% (RAW264.7) and 97.9\% (BM-MSCs), respectively (Supplementary Fig. 9). In addition, lactic dehydrogenase (LDH) released from cells incubated with $\mathrm{Zn}^{2+}$ or BMP-2 modified $\mathrm{TiO}_{2}$ surfaces were detected to determine the cytotoxicity of the materials. After $24 \mathrm{~h}$ incubation, the amount of LDH from these cells was slightly lower than that in the bare $\mathrm{TiO}_{2}$ group, indicating that there was no cytotoxicity (Fig. 3E). These implied the poor biocompatibility of the bare $\mathrm{TiO}_{2}$ surface was significantly improved by $\mathrm{Zn}^{2+}$ modification and BMP-2 peptide conjugation, promising further application in bone-implants. Furthermore, the morphology of adherent BM-MSCs on different surfaces was also investigated to evaluate the surface cytocompatibility. SEM images showed that BM-MSCs on the $\mathrm{TiO}_{2}$ surface exhibited limited cell spreading 

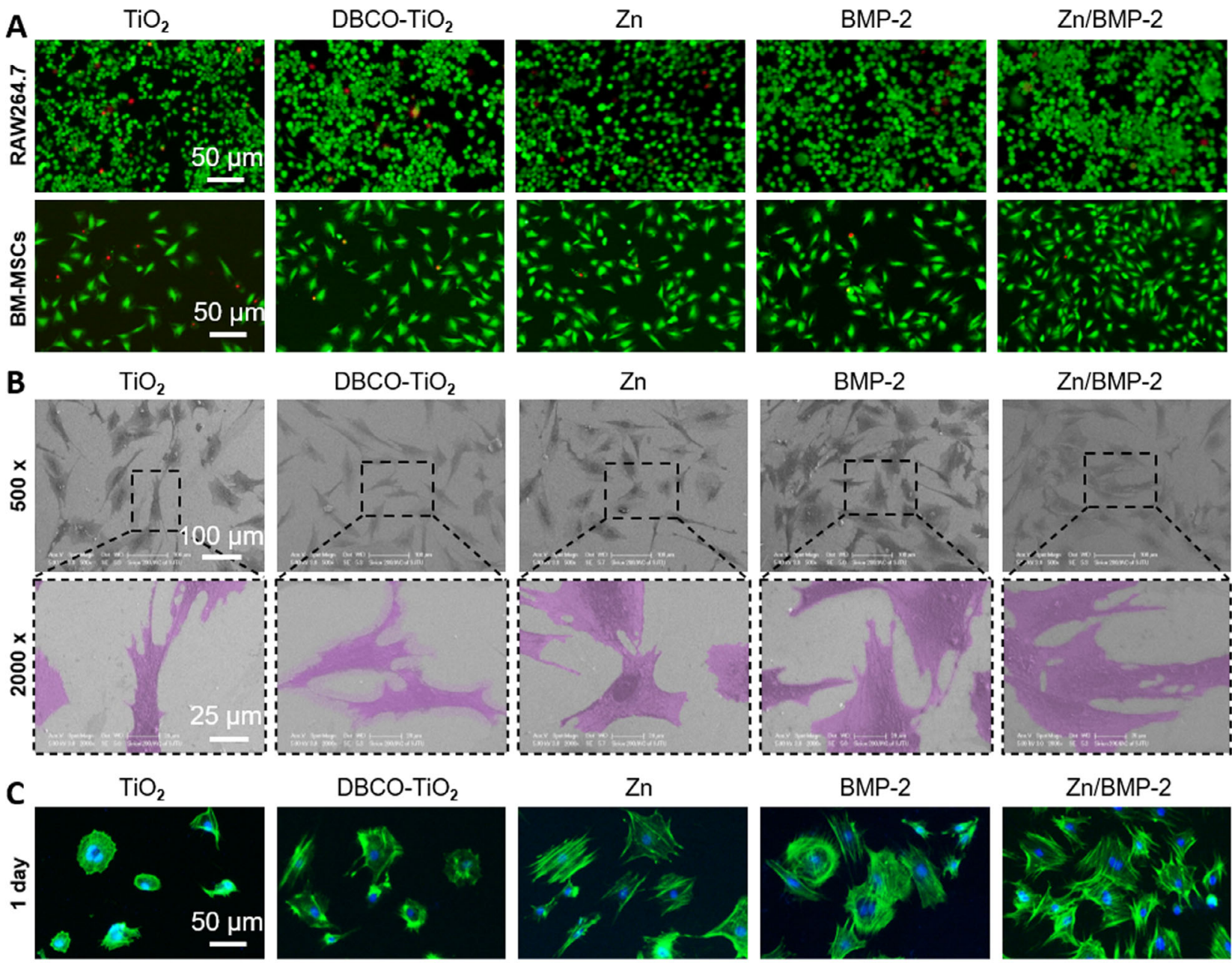

$\mathrm{Zn}$

BMP-2

Zn/BMP-2
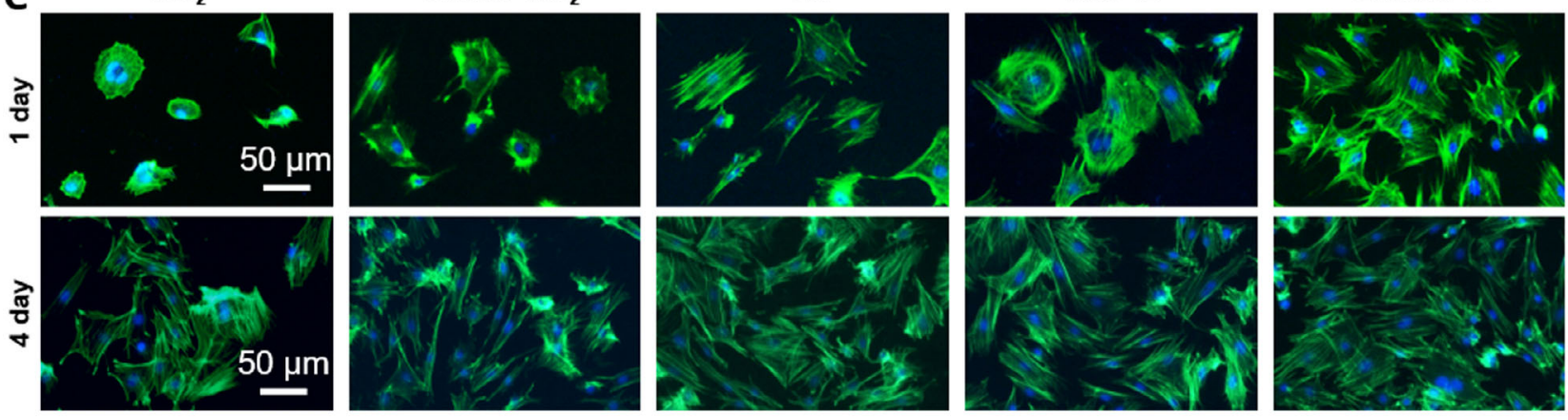

D

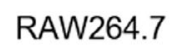

BM-MSCs
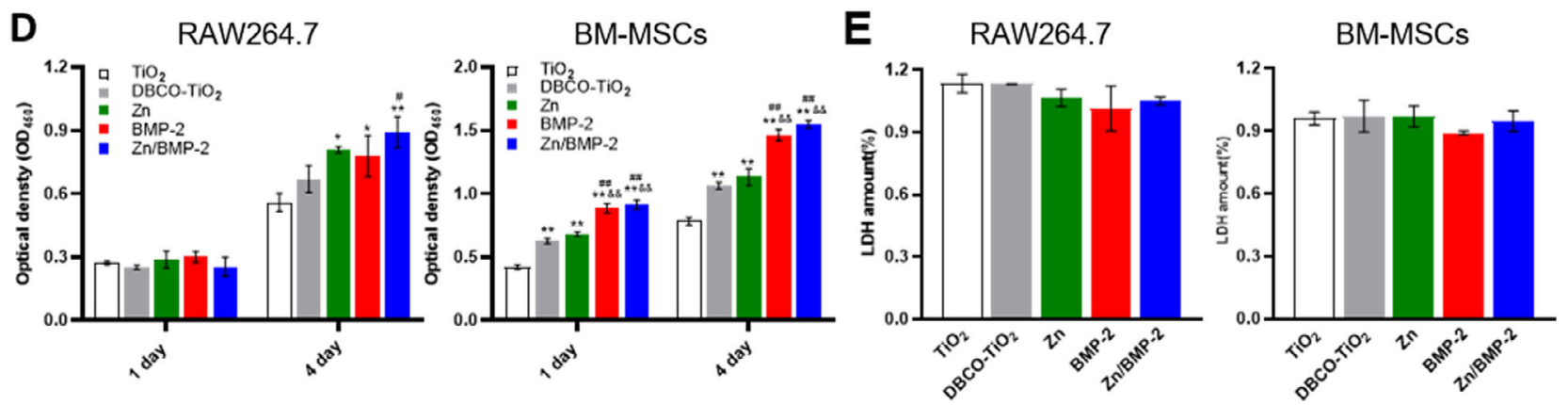

Fig. 3 Biocompatibility properties of $\mathbf{Z n}^{2+}$ and BMP-2 co-modified $\mathbf{T i O}_{\mathbf{2}}$ surfaces. A Live/Dead staining of BM-MSCs and RAW264.7 on the bare and modified $\mathrm{TiO}_{2}$ surface ( $\mathrm{DBCO}-\mathrm{TiO}_{2}, \mathrm{Zn}, \mathrm{BMP}-2$ and $\mathrm{Zn} / \mathrm{BMP}-2$ ) (scar bar $=50 \mu \mathrm{m}$, three independent experiments). B Scanning electron images of BMMSCs on different surfaces ( $\times 500 \mathrm{scar}$ bar $=100 \mu \mathrm{m}$ and $\times 2000 \mathrm{scar}$ bar $=25 \mu \mathrm{m}$, three independent experiments). C The cytoskeleton staining (FITCphalloidin/DAPI) of BM-MSCs on different surfaces for 1 and 4 days (scar bar $=50 \mu \mathrm{m}$, three independent experiments). D Cell viability of BM-MSCs and RAW264.7 on different surfaces for 1 and 4 days (CCK-8) (D $n=3$ independent samples per group, by a one-way ANOVA with a Tukey's post hoc test for multiple comparisons). E Cell cytotoxicity of BM-MSCs and RAW264.7 on different surfaces for $24 \mathrm{~h}$ ( $\mathbf{E} n=3$, independent samples per group, by a oneway ANOVA with a Tukey's post hoc test for multiple comparisons). Data are reported as mean $\pm \mathrm{SD},{ }^{\star} p<0.05$, ${ }^{\star} p<0.01$ compared with the bare $\mathrm{TiO}_{2}$ surface; ${ }_{p} p<0.05,{ }^{\#} p<0.01$ compared with the $\mathrm{DBCO}^{-\mathrm{TiO}_{2}}$ surface; $\&_{p}<0.05, \&_{p}<0.01$ compared with $\mathrm{Zn}$ surface. Exact $P$ values were given in the Source Data file. 
area compared with cells on the $\mathrm{Zn}, \mathrm{BMP}-2$, and $\mathrm{Zn} / \mathrm{BMP}$ 2 surfaces (Fig. 3B). Further studies with cytoskeleton staining (FITC-phalloidin/DAPI) revealed that BM-MSCs on $\mathrm{TiO}_{2}$ exhibited a relatively spherical morphology with almost no filopodia after a 1-day culture. On the contrary, BM-MSCs on the $\mathrm{Zn}, \mathrm{BMP}-2$ and $\mathrm{Zn} / \mathrm{BMP}-2$ surfaces exhibited better cell adhesion with polygonal shapes and high expressions of filamentous F-actin (Fig. 3C). It is noteworthy that there were no obvious differences in cell adhesion and spreading state between all groups after a 4-day culture. Nevertheless, cell counting Kit-8 (CCK-8) assay was then employed to evaluate the proliferation of BM-MSCs and RAW 264.7 cells. The results showed that the surfaces of Zn, BMP-2, and Zn/BMP-2 afforded better cell viability for both RAW 264.7 and BM-MSCs compared with others. Interestingly, the $\mathrm{Zn}^{2+}$-containing surfaces (i.e., the groups of $\mathrm{Zn}$ and $\mathrm{Zn} / \mathrm{BMP}-2$ ) elicited the fastest proliferation of RAW 264.7 cells, while BMP-2 peptide-containing surfaces (i.e., the groups of BMP-2 and $\mathrm{Zn} / \mathrm{BMP}-2$ ) were inclined to enhance BM-MSCs proliferation (Fig. 3D). Impressively, Zn/BMP-2 comodified surface exhibited better cell adhesion, spreading and proliferation, probably due to the synergistic effect of $\mathrm{Zn}^{2+}$ and BMP-2 in promoting the affinity for cells. Taken together, the above results implied that the $\mathrm{Zn}^{2+}$ and BMP-2 peptide comodified surfaces had positive effects on the growth of both macrophages and BM-MSCs with no appreciable cytotoxicity. The reduced cytotoxicity, improved adhesion and proliferation of both the immune cells and multipotential stem cells would be a prerequisite to create a favorable microenvironment for tissue regeneration.

Macrophage phenotypic switching in vitro. The typical immunomodulatory mechanism of biomaterials for tissue repair is to switch the macrophage phenotypes from pro-inflammatory M1 to anti-inflammatory $\mathrm{M}^{21,54,55}$. For bone implants, studies have showed that excessive M1 macrophages can cause bones absorption, which is an important factor leading to implant loosening or implantation failure ${ }^{56,57}$. It has been demonstrated that functional coatings, loaded with cytokines or active ions, could promote macrophage polarization toward an M2 phenotype both in vitro and in vivo and improve the integration of implants into bone tissue ${ }^{24,28}$. Regarding bioactive ions, zinc, an essential trace element for some key enzymes and transcription factors, is considered to be indispensable for the development of the adaptive immune system ${ }^{58,59}$. A clinical study reported that patients with inflammatory diseases (e.g., rheumatoid arthritis, RA) had low serum levels of zinc and corresponding increased levels of pro-inflammatory TNF-a; the process could be reversed by the supplementation of zinc ${ }^{60}$. Besides, some previous reports have already suggested that zinc exerts modulatory effects on macrophage phenotype from M1 to M2, inducing antiinflammatory responses and inhibiting the pro-inflammatory ${ }^{27,28}$. Therefore, the addition of zinc has a potential positive effect on osteoimmunomodulation. In this work, we successfully employed mussel adhesion-mediated ion coordination and molecular clicking strategy to incorporate $\mathrm{Zn}^{2+}$ and BMP-2 peptide onto bone implants. To investigate the effect of our strategy on the regulation of immune microenvironment, the polarization of macrophages on these surfaces was then studied. Macrophage-like cells RAW264.7 in the resting state (M0) were first stimulated by lipopolysaccharide (LPS), and their phenotypes were then evaluated after culture on different surfaces (Fig. 4A). As shown, macrophages (M0) were round, and cells stimulated by LPS developed into the proinflammatory M1 phenotype with a pancake-like shape (Fig. 4B). Due to different surface modifications, the morphology of macrophages on different surfaces showed different states (Fig. 4C). RAW cells on the surfaces without $\mathrm{Zn}^{2+}$ coordination (i.e., the $\mathrm{TiO}_{2}$,
$\mathrm{DBCO}-\mathrm{TiO}_{2}$, and $\mathrm{BMP}-2$ groups) were predominantly pancakeshaped. On the contrary, macrophages switched their morphology into an elongated shape on the $\mathrm{Zn}^{2+}$ modified groups (i.e., the $\mathrm{Zn}$ and $\mathrm{Zn} / \mathrm{BMP}-2$ groups). The significant increase of elongated cells on $\mathrm{Zn}$ and $\mathrm{Zn} / \mathrm{BMP}-2$ surfaces preliminarily indicated the polarization of macrophages from pro-inflammatory M1 phenotype to anti-inflammatory M2 phenotype (Fig. 4D). In addition, to define the shape of macrophages, the degree of cell elongation was further quantified by the ratio of the long axis to the short axis length ${ }^{61,62}$. The macrophages treated with $\mathrm{Zn}^{2+}$-containing coatings showed a significant higher rate of cellular elongation than those on the bare $\mathrm{TiO}_{2}$ surface (control group) (Supplementary Fig. 10). Together, these data suggested that $\mathrm{Zn}^{2+}$-containing surfaces could influence the macrophages morphology and might have an impact on their macrophage phenotypic conversion.

As we know, the M1 and M2 phenotypes have distinguishable markers on cell surfaces and cytokine secretion profiles ${ }^{13,63}$. Thus, the cytokine secretion and the relative expression levels of inflammatory gene markers were determined with enzyme-linked immunosorbent assay (ELISA) and real-time polymerase chain reaction (RT-PCR). Clearly, the secretion of pro-inflammatory cytokine TNF- $\alpha$ in groups of $\mathrm{TiO}_{2}, \mathrm{DBCO}-\mathrm{TiO}_{2}$, and BMP-2 was significantly higher than that in the $\mathrm{Zn}^{2+}$-modified groups ( $\mathrm{Zn}$ and $\mathrm{Zn} / \mathrm{BMP}-2$ ) (Fig. $4 \mathrm{E}$ ), indicating the predominant existence of M1 macrophages on these surfaces. In contrast, the secretion of anti-inflammatory cytokine IL-10 in groups of $\mathrm{Zn}$ and $\mathrm{Zn} / \mathrm{BMP}-2$ efficiently increased, showing potent inflammation-attenuating effect of $\mathrm{Zn}^{2+}$ on macrophage phenotypic switching (Fig. 4F). To further evaluate the macrophages polarization status, the M1 surface markers (CD86 and iNOS) and M2 surface markers (CD206 and Arg-1) in RAW 264.7 macrophages were further labelled by immunofluorescence staining. As shown in Fig. 4G-L, LPS stimulation upregulated the proportion of M1 macrophages (F4/80/CD86 ${ }^{+}$and $\mathrm{F} 4 / 80 / \mathrm{iNOS}^{+}$, red) on the surfaces of $\mathrm{TiO}_{2}$, DBCO-TiO ${ }_{2}$ and BMP-2 groups. In contrast, anti-inflammatory M2 macrophages on the $\mathrm{Zn}$ and $\mathrm{Zn} / \mathrm{BMP}-2$ surfaces were dominant $\left(\mathrm{CD}^{206}{ }^{+}\right.$and $\mathrm{Arg}-\mathrm{1}^{+}$, red). Interestingly, the highest percentage of CD206 and Arg-1 positive cells and the lowest percentage of iNOS and CD86 negative cells were found in the $\mathrm{Zn} / \mathrm{BMP}-2$ group. It was also worth mentioning that the M1 phenotype markers (iNOS and CD86) were slightly downregulated while the the M2 phenotype markers were slightly upregulated in BMP-2 group compared with the controls $\left(\mathrm{TiO}_{2}\right.$ and $\mathrm{DBCO}-\mathrm{TiO}_{2}$ ), probably due to the potential of $\mathrm{BMP}-2$ protein for regulating local osteoimmune microenvironment ${ }^{64}$.

Quantitative RT-PCR analysis also showed similar results. The gene expression levels showed downregulation for proinflammatory TNF- $\alpha$ and upregulation for anti-inflammatory IL-10 in the case of $\mathrm{Zn}^{2+}$ modification, further indicating the potential of $\mathrm{Zn}^{2+}$-modified groups for the switch of macrophage to M2 phenotype (Fig. 4M, N). The expression levels of surface markers associated with M1 (e.g., CCR7) and M2 (e.g., CD206) phenotypes were further determined. The efficiently suppressed expression of $C d 206$ and significantly increased expression of Ccr 7 in $\mathrm{Zn}$ and $\mathrm{Zn} / \mathrm{BMP}-2$ groups further indicated the positive immunomodulatory function of $\mathrm{Zn}^{2+}$-modified groups for M2 phenotype polarization (Fig. 4O, P). It is interesting to note that the above results all reflected the $\mathrm{Zn}^{2+}$ and BMP-2 peptide comodified surface elicited the most efficient M2 phenotype polarization, probably due to the potential immunomodulatory role of BMP-2 peptide which may motivate the immunoactivity of $\mathrm{Zn}^{2+64}$. In addition, the gene expression levels of osteogenic cytokines secreted from the M2 macrophages (e.g., Bmp-2 and $V e g f)$ were also upregulated on the $\mathrm{Zn}^{2+}$-containing surfaces, with the highest expression in the $\mathrm{Zn} / \mathrm{BMP}-2$ surface (Fig. $4 \mathrm{Q}, \mathrm{R}$ ). The $\mathrm{Zn}^{2+}$-enhanced osteogenic cytokine secretion together with 
A

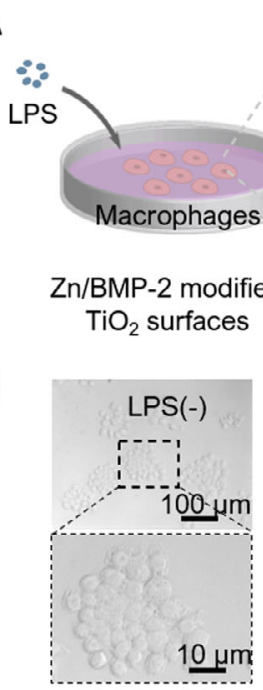

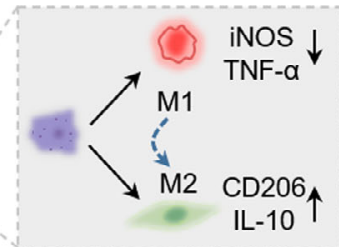

Switching M1 to M2 macrophages phenotypic
C

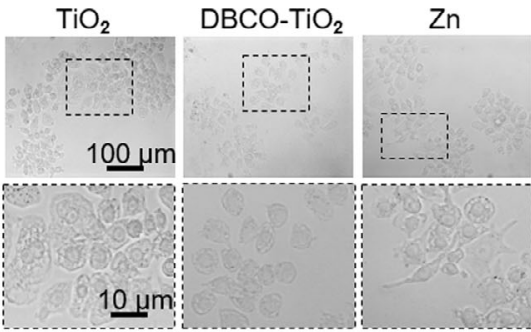

BMP-2

Zn/BMP-2

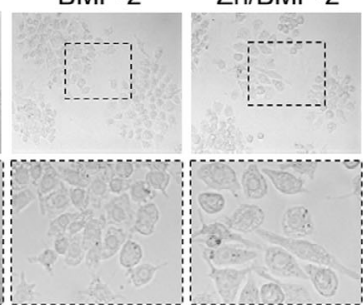

\section{B}
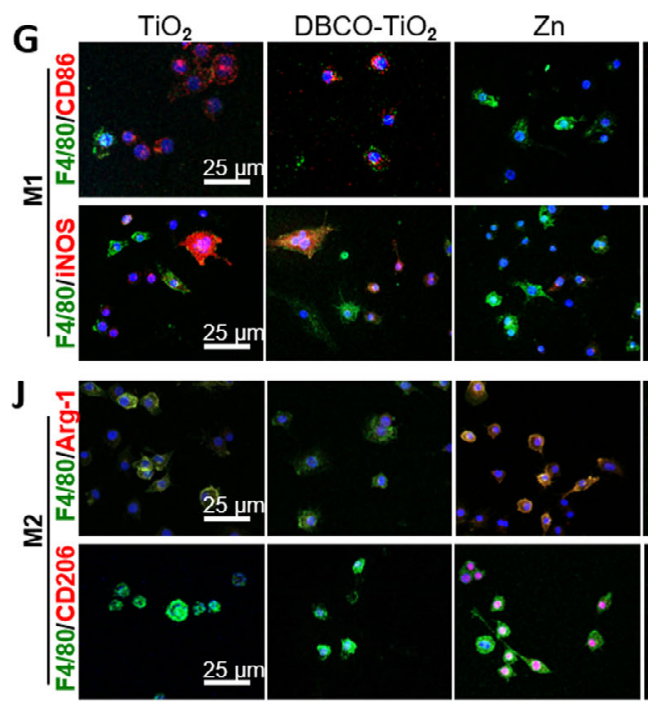

D

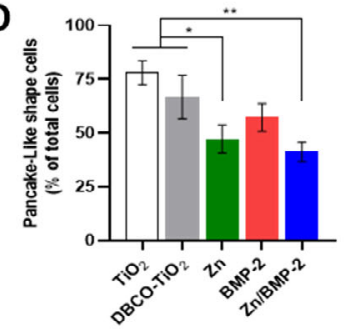

E

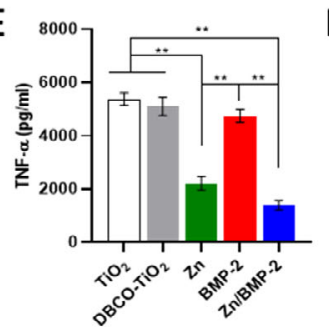

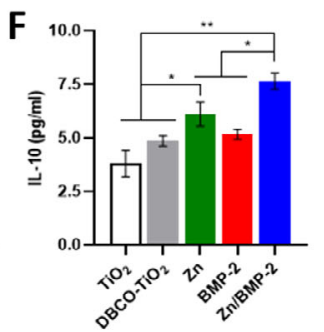
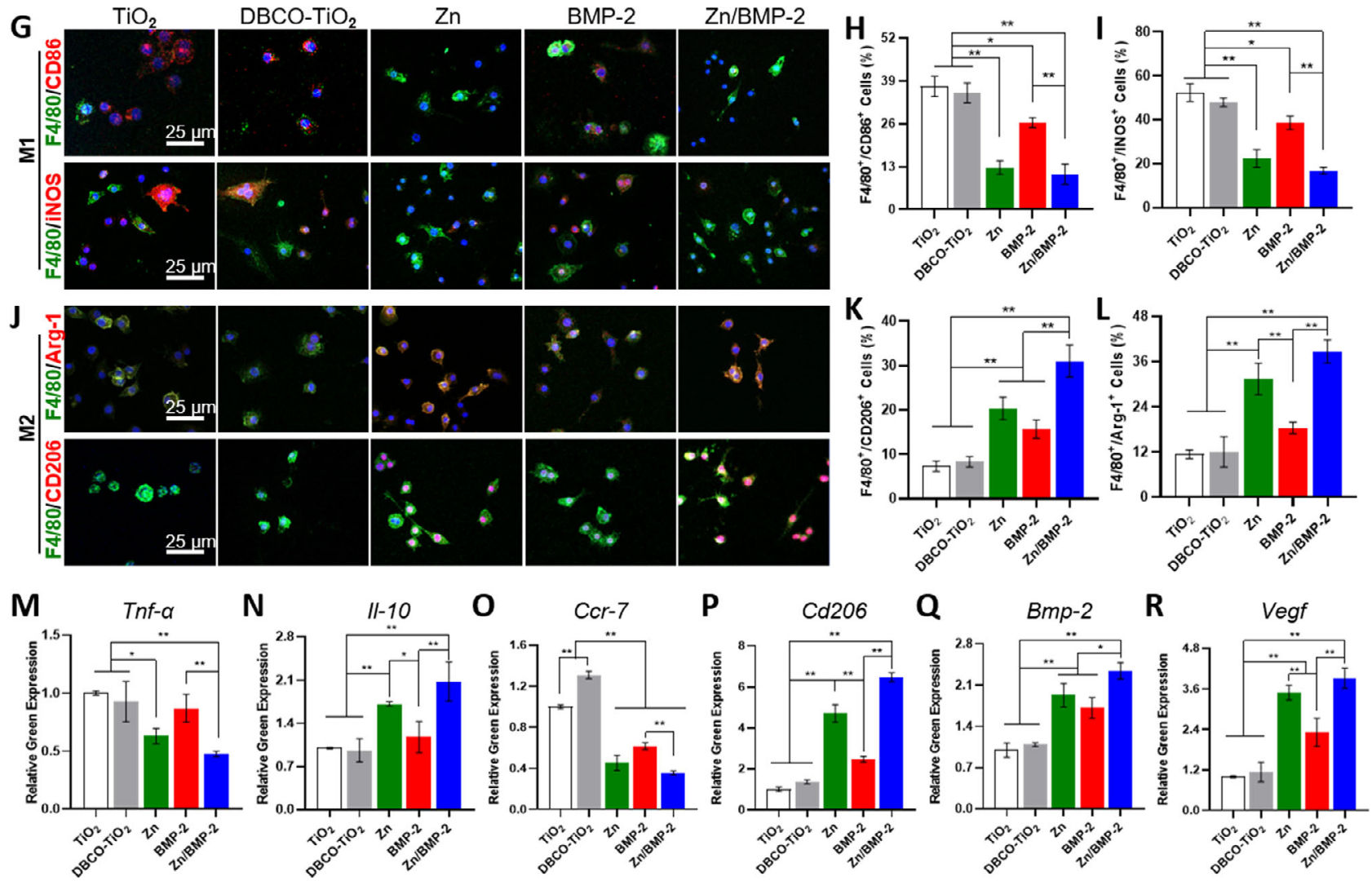

Fig. $4 \mathrm{Zn}^{2}+$ and $\mathrm{BMP-2}$ peptide co-modified $\mathrm{TiO}_{2}$ surface regulate macrophages polarization in vitro. A The illustration of experimental design; $\mathbf{B}$ The morphology of RAW246.7 was stimulated by LPS or without LPS; C The morphology of RAW264.7 was cultured on different surfaces $\left(\mathrm{TiO}_{2}\right.$, DBCO-TiO ${ }_{2}$ $\mathrm{Zn}, \mathrm{BMP}-2$, and Zn/BMP-2 surface) (scar bar $=100 \mu \mathrm{m}$ and $10 \mu \mathrm{m}$, three independent experiments) and (D) Quantitative results of pancake-like shape cells (M1) as a proportion of total cells ( $\mathbf{D} n=3$ biologically independent samples per group, by a one-way ANOVA with a Tukey's post hoc test for multiple comparisons); E TNF- $\alpha$ and (F) IL-10 cytokine secretion by ELISA (E-F $n=3$ biologically independent samples per group, by a one-way ANOVA with a Tukey's post hoc test for multiple comparisons). G, J Immunofluorescent staining results for RAW264.7 cultured on different surfaces: red (M1 marker: CD86 or iNOS and M2 marker: CD206 or Arg-1), green (F4/80, a monoclonal antibody specifically directing against the mouse macrophage), and blue (nuclei) (scar bar $=25 \mu \mathrm{m}$, three independent experiments); Corresponding percentage of double-positive macrophages M1 (H, I) and M2 (K, L) (H-I, K-L $n=3$ biologically independent samples per group, by a one-way ANOVA with a Tukey's post hoc test for multiple comparisons); M-R RT-PCR results of Tnf- $\alpha$, II-10, C Cr7, Cd206, Bmp-2, and Vegf respectively (M-R $n=3$, biologically independent samples per group, by a one-way ANOVA with a Tukey's post hoc test for multiple comparisons). Data are reported as mean $\pm \mathrm{SD},{ }^{\star} p<0.05,{ }^{\star \star} p<0.01$. Exact $P$ values were given in the Source Data file. 
the BMP-2 peptide-enhanced immunomodulatory function collectively revealed the different but overlapping roles of $\mathrm{Zn}^{2+}$ and BMP-2 peptide in immunomodulation and osteoinduction. Therefore, their combination (i.e., Zn/BMP-2) may provide a more favorable microenvironment and create improved bone repair potentials at bone-to-implant interfaces.

Although zinc plays an important and beneficial role in immune functions, its effects depend on the concentration of zinc $^{58}$. A study reported that $1.25 \times 10^{-6} \mathrm{M}(0.08 \mathrm{ppm})$ zinc was enough to inhibit the expression of pro-inflammatory cytokines; however, a low concentration of $\mathrm{Zn}^{2+}(<6.5 \mathrm{ppm})$ had no osteoclast activity, while higher $\mathrm{Zn}^{2+}$ amounts increased osteoclast activity ${ }^{65}$. In the present study, zinc concentration reached $0.145 \mathrm{ppm}$ on the first day, and the total zinc ion released from $\mathrm{Zn} / \mathrm{BMP}-2$ dual-effect coating was $0.575 \mathrm{ppm}$ after 28 days (Fig. 2O), which is much lower than $6.5 \mathrm{ppm}$. Furthermore, zinc ions at the concentration of $0.145 \mathrm{ppm}$ could elevate the proliferation of both macrophages and BM-MSCs (Fig. 3), activate the macrophage phenotypic switch from M1 to M2 (Fig. 4) and enhance the osteogenic differentiation (Fig. 5).

Immunomodulation-enhanced osteogenic differentiation in vitro. A larger number of cells, including immune cells and BM-MSCs, are recruited to the bone implant's surface after implantation. The osteogenic differentiation of BM-MSCs in vivo is regulated by not only surface properties of implants but also surrounding immune microenvironment. Accordingly, the immunomodulatory effect of $\mathrm{Zn} / \mathrm{BMP}-2$ surface on in vitro osteogenic differentiation was further investigated by using macrophage conditioned medium (MCM $)^{20,27,28}$ (Fig. 5A). At day 14, the expressions of three osteogenic-related proteins, including ALP, calcium binding proteins and osteopontin (OPN), were determined to evaluate the efficiency of osteogenic differentiation. The ALP staining clearly showed that significantly higher ALP activity was detected in the MCMs derived from $\mathrm{Zn}^{2+}$ or BMP-2 peptide modified surfaces (i.e., the $\mathrm{Zn}, \mathrm{BMP}-2$, and $\mathrm{Zn} / \mathrm{BMP}-2$ groups) compared with the $\mathrm{TiO}_{2}$ and DBCO$\mathrm{TiO}_{2}$ group (Fig. 5B). Quantitative analysis of ALP staining revealed that the ALP activity in the $\mathrm{Zn} / \mathrm{BMP}-2$ derived MCM were $5.06,4.22,2.00$, and 1.80-fold higher than that of $\mathrm{TiO}_{2}$, DBCO-TiO $2, \mathrm{Zn}$ and BMP-2 groups, respectively (Fig. 5E, F). Meanwhile, ALP immunofluorescence images further confirmed the results that the $\mathrm{Zn} / \mathrm{BMP}-2$ derived MCM could induce the highest level of ALP activity (Supplementary Fig. 11), indicating the $\mathrm{Zn} / \mathrm{BMP}-2$ co-modified surface could regulate the immunoregulatory effect on osteogenic differentiation of BM-MSCs. In addition to ALP protein level, similar trends were also found in the expressions of other osteogenesis-related proteins (calcium binding proteins and OPN). For example, Alizarin Red S (ARS) staining for calcium binding proteins in the mineralized matrix at day 14 showed that the size and quantity of the mineral nodules in $\mathrm{Zn} / \mathrm{BMP}-2$ group was 1.50 and 1.33-fold higher than that of the $\mathrm{Zn}$ and BMP-2 groups, indicating the most efficient calcium deposition in BM-MSCs cultured with $\mathrm{Zn} / \mathrm{BMP}-2$ derived MCM (Fig. 5C-G). OPN immunofluorescence staining also confirmed the most efficient enhancement of osteogenic differentiation in $\mathrm{Zn} / \mathrm{BMP}-2$ derived MCM, in which the relative OPN expression increased by $46.10 \%$ and $34.11 \%$ compared with the $\mathrm{Zn}$ and BMP-2 groups, respectively. Apart from the above osteogenesisrelated proteins, we also investigated the expression levels of osteogenesis-related genes, including $A l p$, runt-related transcription factor 2 (Runx2), type I collagen (Colla1) and Opn (Fig. 5I-L). As expected, the mRNA expressions of these osteogenesis-related genes in BM-MSCs cultured with Zn/BMP-2 derived MCM were all significantly higher than others, confirming the potent ability of Zn/BMP-2 derived MCM to enhance osteogenic differentiation. Overall, these results demonstrated that the combination of immunoactive $\mathrm{Zn}^{2+}$ and osteoinductive BMP-2 peptide would be more conducive to the regulation of macrophage phenotypic switch from $\mathrm{M} 1$ to $\mathrm{M} 2$, and the secreted cytokines would provide an optimum osteoimmunomodulatory microenvironment and lead to immuno-enhanced osteogenesis.

Macrophage phenotypic switching in vivo. Macrophages play a pivotal role in osteoimmunomodulation due to their plasticity and direct function in the inflammatory process of early neo-bone formation. A switch to the M2 phenotype has been shown to be essential for bone healing and osteointegration around implants ${ }^{17}$. In this study, the above in vitro studies have already demonstrated that the Zn/BMP-2 co-modified surface possessed excellent properties including improved cytocompatibility to accelerate cell growth, efficient macrophage phenotype regulation from $\mathrm{M} 1$ to $\mathrm{M} 2$, and potent osteoimmunomodulatory activity to enhance osteogenicity. These advantages guide us to further investigate the immunomodulatory activity and osteogenicity in vivo. A commercially available cortical bone self-tapping Tibased screw was used as the model implant. The screws were treated the same as that of $\mathrm{TiO}_{2}$-deposited substrates to obtain different surface modification (named as $\mathrm{DBCO}-\mathrm{TiO}_{2}, \mathrm{Zn}, \mathrm{BMP}$ 2 and $\mathrm{Zn} / \mathrm{BMP}-2$, respectively). The untreated Ti screw, marked as the control group in vivo, was also named as $\mathrm{TiO}_{2}$ owing to the tight and continuous $\mathrm{TiO}_{2}$ layer on it. Screw implantation in the femoral condyles of rats was then performed according to standard surgical protocol. To minimize the damage to surface layer during implantation, a drill with a diameter between the concave and convex thread of screw was used in animal experiments (the yellow line in Supplementary Fig. 12). 4 days after implantation, the rat femoral condyles containing the implanted Ti screws were harvested for histological hematoxylin-eosin (H\&E) and Goldner's trichrome staining. H\&E images showed that the tissues near the screws in the groups of $\mathrm{Zn}, \mathrm{BMP}-2$, and $\mathrm{Zn} / \mathrm{BMP}-2$ exhibited milder inflammatory response, thinner fibrous layers and more complete bone structures as compared with that of the $\mathrm{TiO}_{2}$ and $\mathrm{DBCO}-\mathrm{TiO}_{2}$ groups (Fig. 6A). The thinnest fibrous layer was observed in the $\mathrm{Zn} / \mathrm{BMP}-2$ group, while the milder inflammatory response in $\mathrm{Zn}$ and $\mathrm{Zn} / \mathrm{BMP}-2$ screws were comparable (Fig. 6D, E). Goldner's trichrome analysis highlighted the calcified bone (green) significantly increased at the implantation site of $\mathrm{Zn} / \mathrm{BMP}-2$ co-modified group in comparison with others, indicating a higher extent of integration and a larger amount of newborn trabecular structures adjacent to the implant (Supplementary Fig. 13). These results implied that the Zn/BMP-2 comodified screw could create a favorable immune microenvironment for enhancing osteointegration between bone tissues and implants. It was worth mentioning that a slightly less improvement on inhibition of fibrosis and inflammatory response was shown in the BMP-2 group, still indicating the potential immunoactive of the BMP-2 peptide ${ }^{64}$. On the contrary, the thick fibrous layers and large-scale infiltration of inflammatory cells around the bare screw indicate the reason of most implantation failures. In addition, the phenotypic conversion of macrophages around the screws was further evaluated with immunofluorescence staining. Inflammatory cell infiltration was determined by labelling CD68, and the M1 to M2 phenotypic switching was determined by labelling CCR7 and CD206-positive cells, respectively (Fig. 6B). Immunofluorescence images showed CD206-positive cells (i.e., M2 phenotypic macrophages) around $\mathrm{Zn}, \mathrm{BMP}-2$ and $\mathrm{Zn} / \mathrm{BMP}-2$ screws were significantly more than those around the control screws in $\mathrm{TiO}_{2}$ and $\mathrm{DBCO}-\mathrm{TiO}_{2}$. 
A

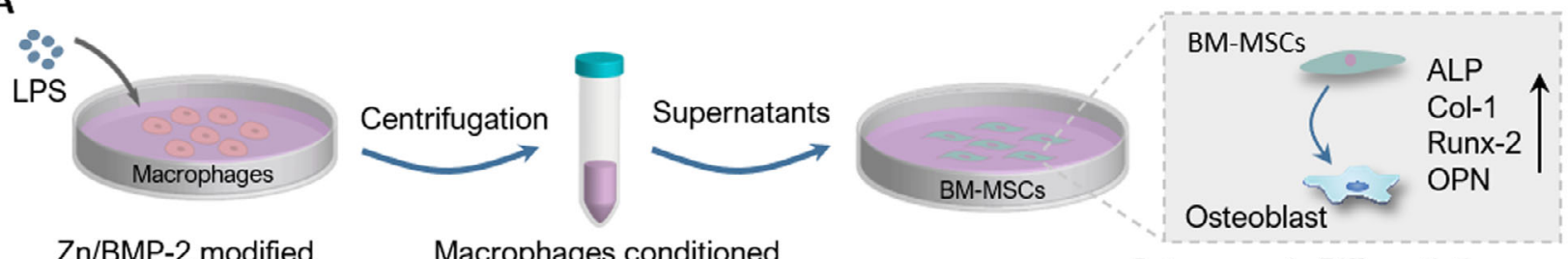

Zn/BMP-2 modified Macrophages conditioned $\mathrm{TiO}_{2}$ surfaces medium (MCM)

Osteogenesis Differentiation

B

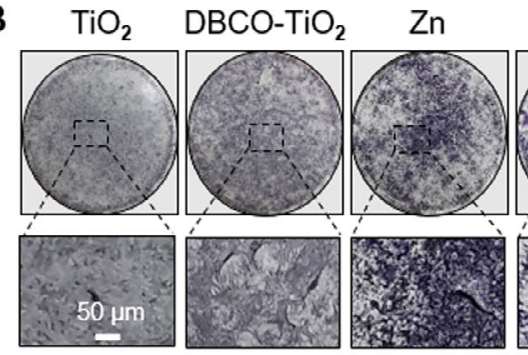

C

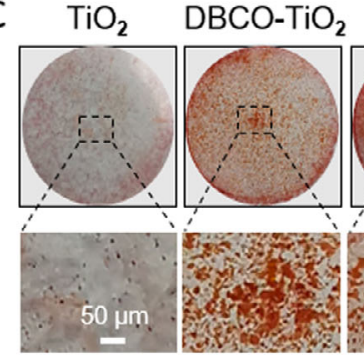

$\mathrm{Zn}$
BMP-2 Zn/BMP-2

BMP-2 Zn/BMP-2
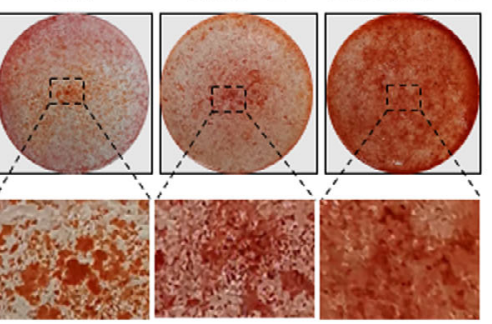

$\mathbf{E}$

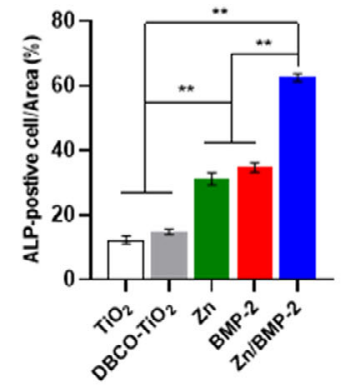

F

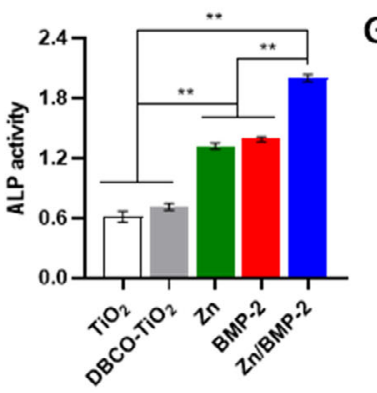

I

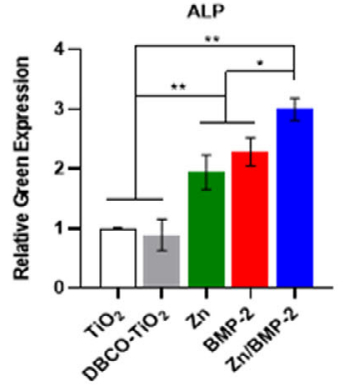

J

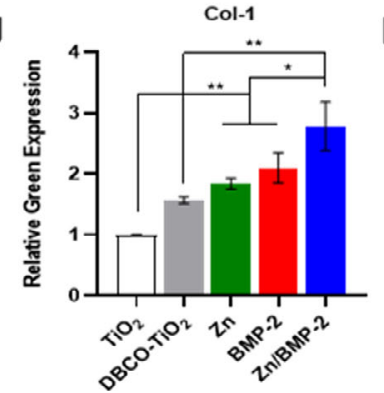

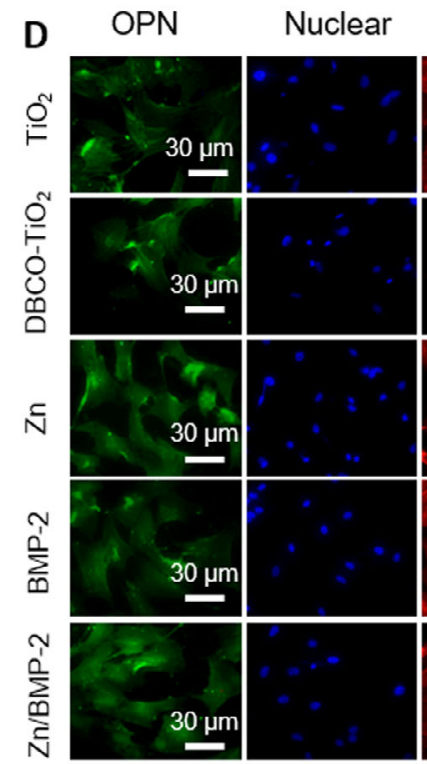

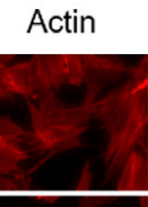

Merged
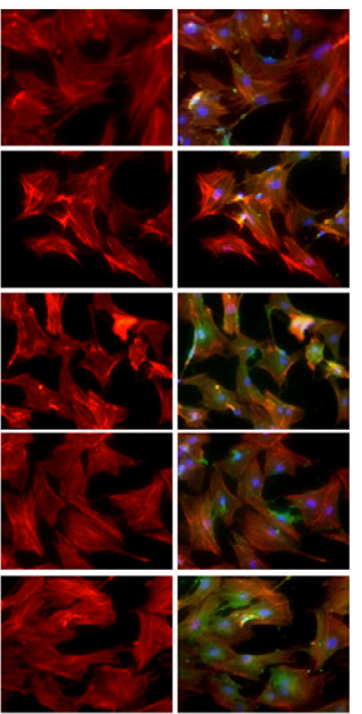

G

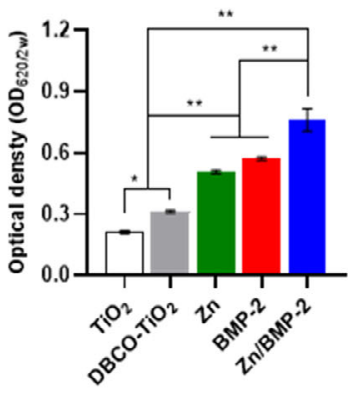

H

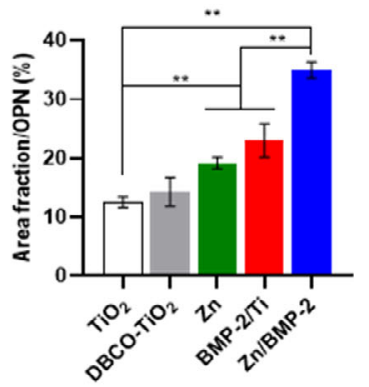

K

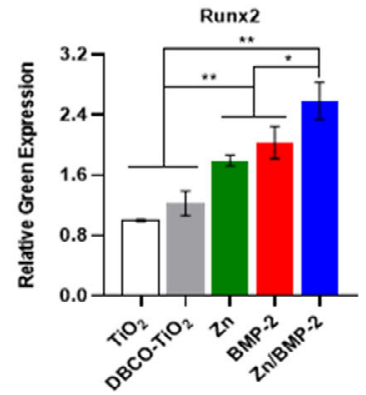

L

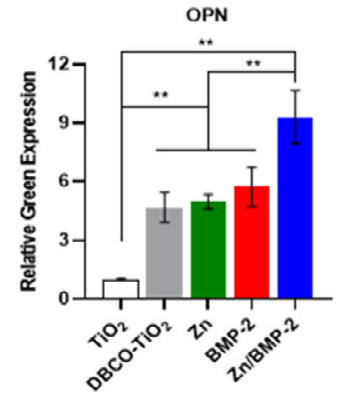

Fig. $5 \mathrm{Zn}^{2}+$ and $\mathrm{BMP-2}$ peptide co-modified $\mathrm{TiO}_{2}$ surfaces enhance osteogenic differentiation in vitro. A The illustration of experimental design; B, E ALP staining and $(\mathbf{C}, \mathbf{G})$ ARS staining of BM-MSCs cultured in osteogenic medium supplemented with MCM (B-C scar bar $=50 \mu \mathrm{m}$, three independent experiments); F ALP activity assay of the BM-MSCs on the different surfaces; $\mathbf{D}$ Images of the BM-MSCs after immunofluorescent staining:(green: OPN; red: cytoskeleton and blue: nuclei) (scar bar $=30 \mu \mathrm{m}$, three independent experiments) and (H) quantitative results; I-L Osteogenesis-related genes expression of the BM-MSCs cultured in MCM detected by RT-PCR (Alp, Runx2, Col1a1 and Opn). (E-L $n=3$ biologically independent samples per group, by a one-way ANOVA with a Tukey's post hoc test for multiple comparisons). Data are reported as mean \pm SD, ${ }^{*} p<0.05$, ${ }^{\star \star} p<0.01$. Exact $P$ values were given in the Source Data file. 

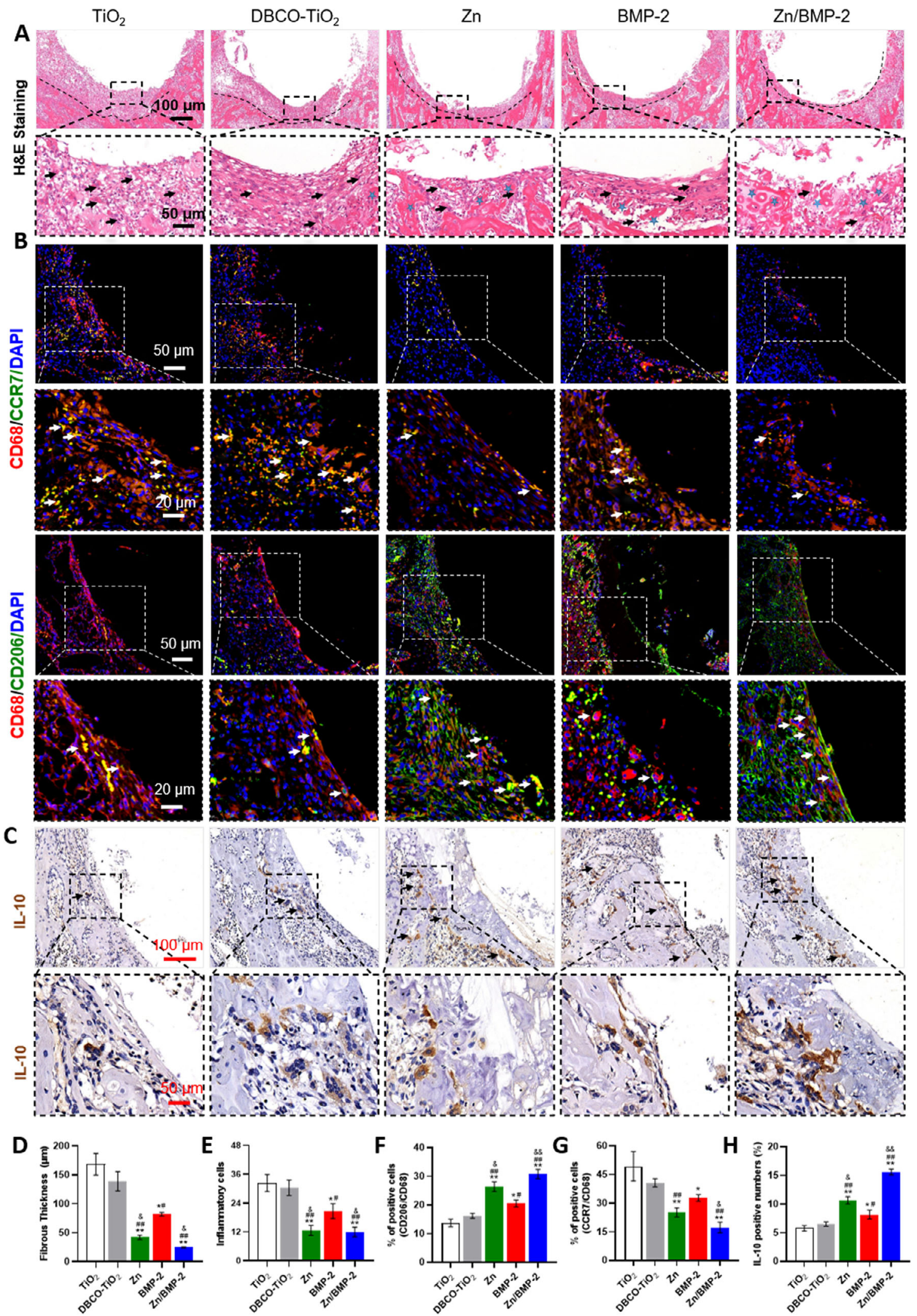

Quantitative analysis showed that the proportion of M2 macrophages in $\mathrm{Zn}, \mathrm{BMP}-2$ and $\mathrm{Zn} / \mathrm{BMP}-2$ groups was about 1.92, 1.50, and 2.25 folds higher than that in the $\mathrm{TiO}_{2}$ group, respectively (Fig. 6F). In contrast, the number of CCR7-postive cells (M1 phenotypic macrophages) in the $\mathrm{TiO}_{2}$ and $\mathrm{DBCO}-\mathrm{TiO}_{2}$ groups was higher than that in others, with the lowest number of M1 macrophages in the Zn/BMP-2 group (Fig. 6G). Immunohistochemical staining further revealed that the deposition of antiinflammatory cytokine IL-10 dramatically increased in the $\mathrm{Zn}$ (10.63\%), BMP-2 (8.16\%), and Zn/BMP-2 (15.61\%) groups as compared with the $\mathrm{TiO}_{2}$ control $(5.76 \%)$ (Fig. 6C-H). This result, together with the reduced number of infiltrated macrophages and 
Fig. $6 \mathrm{Zn}^{2}+$ and BMP-2 peptide co-modified Ti screws regulate macrophages polarization in vivo. A H\&E staining images of the peri-implant tissue (scar bar $=100 \mu \mathrm{m}$ and $50 \mu \mathrm{m}$, three independent experiments) and quantified with (D) fibrous layers and (E) infiltration inflammatory cells; B Coimmunostaining images of the peri-implant tissue: green (M1 marker, CCR7 and M2 marker, CD206), red (CD68, rat macrophage-specific antigen marker), and blue (nuclei) with white arrows indicating the double-positive cells (scar bar $=50 \mu \mathrm{m}$ and $20 \mu \mathrm{m}$, three independent experiments) and (F, G) Quantitative double-positive macrophages; C Images of immunohistochemical staining of IL-10 in the peri-implant tissue (scar bar $=100 \mu \mathrm{m}$ and $50 \mu \mathrm{m}$, three independent experiments) and (H) quantification of IL-10 positive cells as a proportion of total cells. (D-H $n=3$ biologically independent samples per group, by a one-way ANOVA with a Tukey's post hoc test for multiple comparisons). Data are reported as mean \pm SD, ${ }^{\star} p<0.05,{ }^{\star \star} p<0.01$ compared with the bare $\mathrm{TiO}_{2}$ surface; ${ }^{\#} p<0.05,{ }^{\#} p<0.01$ compared with the $\mathrm{DBCO}-\mathrm{TiO}_{2}$ surface; $\&_{p}<0.05, \& \varepsilon_{p}<0.01$ compared with BMP-2 surface. Exact $P$ values were given in the Source Data file.

increased conversion of M1 to M2 phenotypes, confirmed the most potent immunomodulatory activity of $\mathrm{Zn} / \mathrm{BMP}-2$ comodified screw in vivo and implied its potential to reverse the excessive inflammation caused by injury and enhance interfacial osseointegration around the implants.

Osseointegration in vivo. As we know, successful osteointegration requires a favorable immune microenvironment, which promotes osteogenesis differentiation and neo-bone formation around the implants ${ }^{21}$. Osteoimmunomodulation determines the capacity of implantable biomaterials on modulating the osteoimmune environment, thus regulating neo-bone formation. As we have verified that the $\mathrm{Zn} / \mathrm{BMP}-2$ surface possessed the optimal immunoactivity to regulate macrophage M1/M2 polarization in vitro and in vivo and significantly enhanced osteogenesis in vitro, the new bone formation and osseointegration at the screw-to-bone interface was further evaluated 8 weeks after implantation. The in vivo toxicity reaction of the implanted screws on heart, liver, spleen, lung, and kidney were first evaluated, and no significant tissue toxicity was observed, indicating the cytocompatibility of our strategy (Supplementary Fig. 14). Then, Micro-CT 3D reconstruction and histological analysis of the harvested bone tissues with screws were performed. The reconstructed micro-CT $3 \mathrm{D}$ images showed that the highest amount of newly formed bone tissue around the screws was observed in the Zn/BMP-2 group, while few disconnected bone tissues were found in the $\mathrm{TiO}_{2}$ control (Fig. 7A). Quantitative analysis further confirmed this result. Tissues in the $\mathrm{Zn} / \mathrm{BMP}-2$ group had the highest percentage of bone mineral density (BMD) and bone volume to tissue volume (BV/TV) and exhibited the best trabecular structural features of the new bone under the same volume of interest (VOI) (Fig. 7B). The value of BV/TV was 1.37 and 1.26-fold higher in the co-modified $\mathrm{Zn} / \mathrm{BMP}-2$ group $(82.06 \pm 1.46 \%)$ as compared to that in $\mathrm{Zn}(59.77 \pm 3.89 \%)$ and BMP-2 groups $(65.35 \pm 3.63 \%)$, respectively. In addition, the values of trabecular separation ( $\mathrm{Tb} . \mathrm{Sp}$ ), trabecular thickness (Tb. $\mathrm{Th}$ ), and trabecular number ( $\mathrm{Tb} . \mathrm{N}$ ) in the $\mathrm{Zn} / \mathrm{BMP}-2$ group were $70.92 \%, 128 \%$, and $162 \%$ of that in $\mathrm{Zn}$ group whereas $84.28 \%$, $137 \%$, and $143 \%$ of that in BMP-2 group, respectively. As expected, the Zn/BMP-2 co-modified surface showed the best osseointegration between bone tissue and implants, probably due to the synergy of immunoactive $\mathrm{Zn}^{2+}$ ion and osteoinductive BMP-2 peptide, while a mono-modification (e.g., the Zn or BMP2 group) might not provide the most favorable immunomodulatory microenvironment for bone regeneration. Sequential fluorescence labelling was further performed by using Calcein (green) and Alizarin Red (red) to mark the newly formed bone, and similar results were obtained (Fig. 7C, D). A large area of new bone mineralization on the screw surface was observed in $\mathrm{Zn}$ / BMP-2 group (18.80\%), while a gradually decreased bone mineralization was found in the groups of BMP-2 (11.10\%), $\mathrm{Zn}$ (8.22\%), DBCO- $\mathrm{TiO}_{2}(4.20 \%)$ and $\mathrm{TiO}_{2}$ (3.74\%). Quantitative analysis showed that the bone-implant contact ratio (BIC) of $\mathrm{Zn} /$
BMP-2 group was significantly higher (more than twofolds in the value) than the other groups (Fig. 7E).

Since the stable connections between the implants and surrounding bone tissue are closely related to the clinical outcomes of implantation, a biomechanical pull-out experiment was then applied to test the anchorage force of $\mathrm{Ti}$ screws in bone tissue. As shown in Fig. 7F and Supplementary Fig. 15, the maximal pull-out forces in the Zn, BMP-2, and Zn/BMP-2 groups all significantly improved as compared with that of the $\mathrm{TiO}_{2}$ control, indicating the excellent mechanical stability. To be exact, the highest maximum pull-out force $(203.3 \pm 14.3 \mathrm{~N})$ was found in the $\mathrm{Zn} / \mathrm{BMP}-2$ group, which was nearly 2.1 -fold higher than that of the $\mathrm{TiO}_{2}$ control $(98.6 \pm 16.0 \mathrm{~N})$. These results confirmed that the $\mathrm{Zn} / \mathrm{BMP}-2$ surface could significantly promote interfacial osteogenesis and enhance osseointegration in vivo. Overall, we verified that both the immunomodulatory function and direct osteogenicity were crucial to bone implants, according to the overlapping but distinct stages in tissue regeneration (i.e., immune responses and healing processes). In this work, an osteoinductive and immunomodulatory dual-effect implant was readily obtained by co-modifying immunoactive $\mathrm{Zn}^{2+}$ and osteoinductive BMP-2 peptide with a mussel adhesion-mediated ion coordination and molecular clicking method. The dual-effect implant enabled not only M2 phenotypic switching but also direct osteoinductivity, which synergistically created a favorable microenvironment in vivo for bone regeneration (Fig. 7G).

Up to now, no relevant study has successfully combined metal ions and growth factors, and the synergistic effect on osteogenic differentiation or osteointegration is unclear. In this study, we reported on the use of a mussel-like surface coating with immobilized immunomodulatory metal ions (e.g., $\mathrm{Zn}^{2+}$ ) and osteoinductive growth factors (e.g., BMP-2-derived peptide), and we demonstrated the improved in vivo outcomes. Zn/BMP-2 comodified Ti implants increased bone formation by up to $80 \%$ at 8 weeks, which was significantly higher than either stimulator alone 66,67 . For example, some biomimetic implants have improved bone formation (BV/TV) by $28.8 \%$ in vivo ${ }^{66}$ at 4 weeks via $\mathrm{Zn}^{2+}$ delivery; BMP-2 delivery strategies have likewise increased bone formation by $55 \%{ }^{67}$. The possible reasons are as follows. Zinc has been delivered as an acute released ion that diffuses to act on cells (e.g., immune cells) distant from the implant, providing a favorable immunomodulatory microenvironment. Besides, BMP-2 peptide has been fixed to the implants chemically, so side effects associated with unexpected changes in release rates are prevented ${ }^{68,69}$, and BMP-2 peptide will only act upon recruited/activated cells (e.g., osteogenic cell) that contact implant surfaces directly, therefore improving the osseointegration at bone-to-implant interfaces.

The outlook of this study is that it provides a novel solution in a dual-functional implants with both osteoinductive and immunomodulatory activity for improving osseointegration by a mussel adhesion-mediated ion coordination and molecular clicking strategy to effectively improve mechanical fixation of the bone implants. This strategy involves combining the metal ion 
A

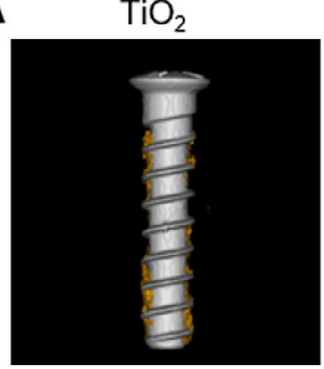

B

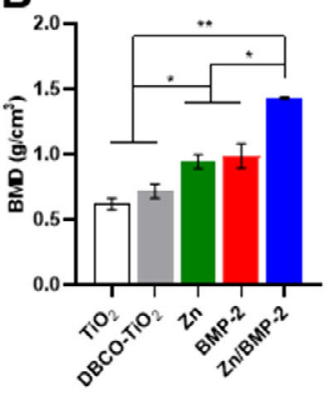

C
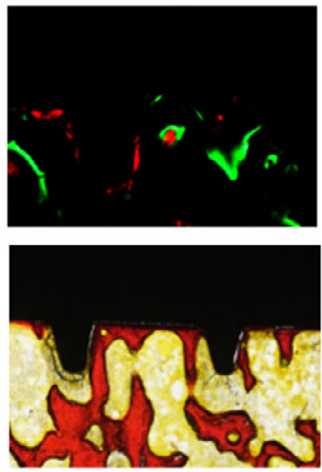

D

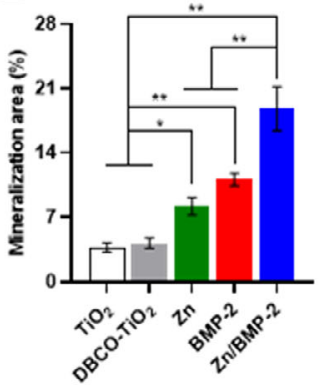

DBCO-TiO
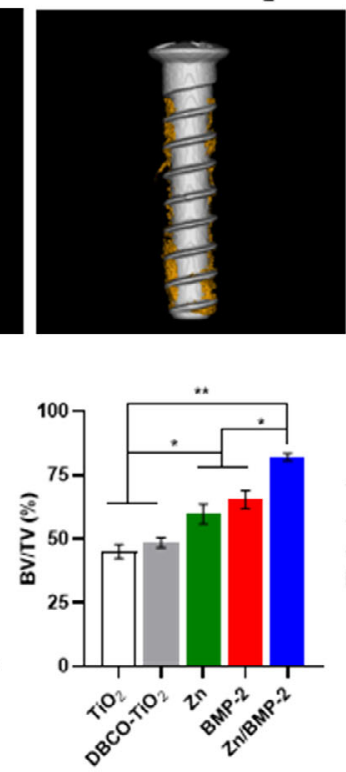

DBCO-TiO
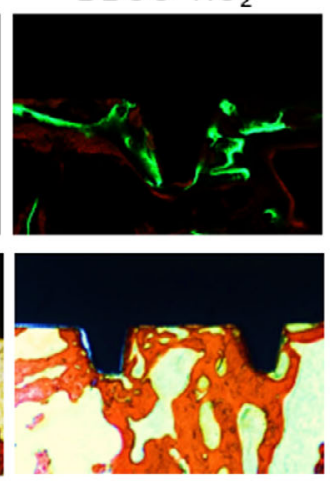

$E$

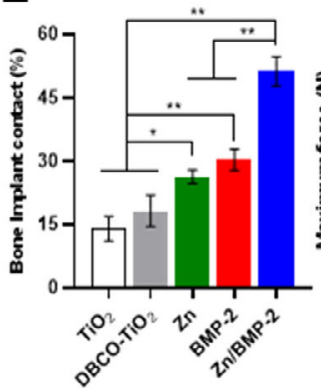

$\mathrm{Zn}$
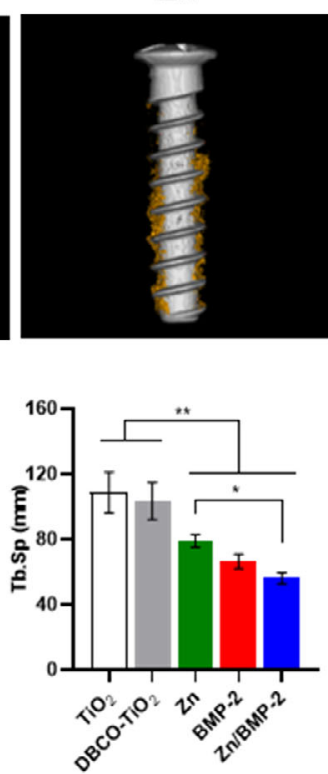

Zn
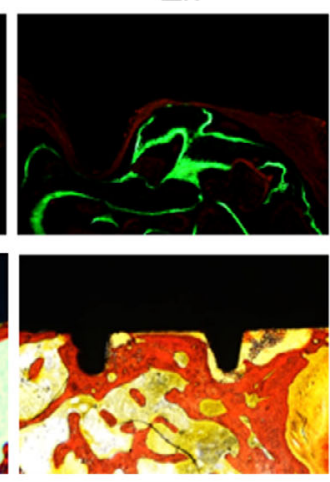

$\mathbf{F}$

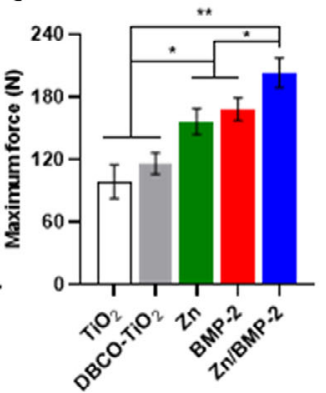

BMP-2
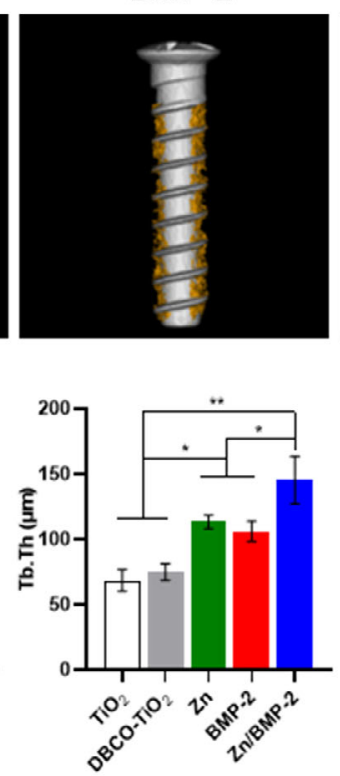

BMP-2
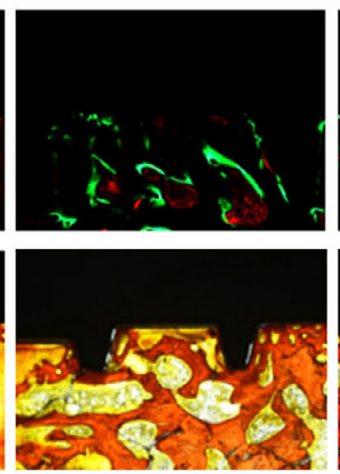

G

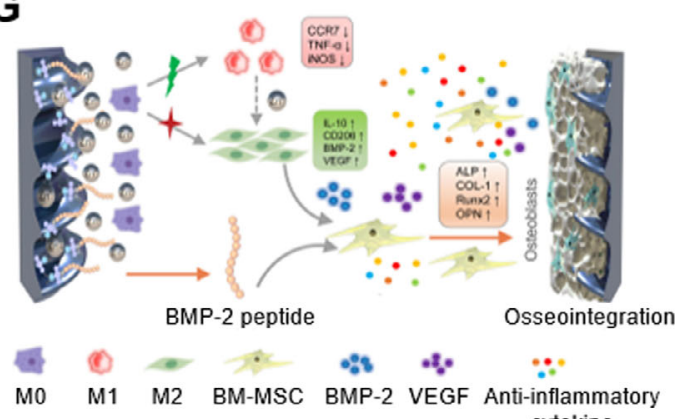

Zn/BMP-2
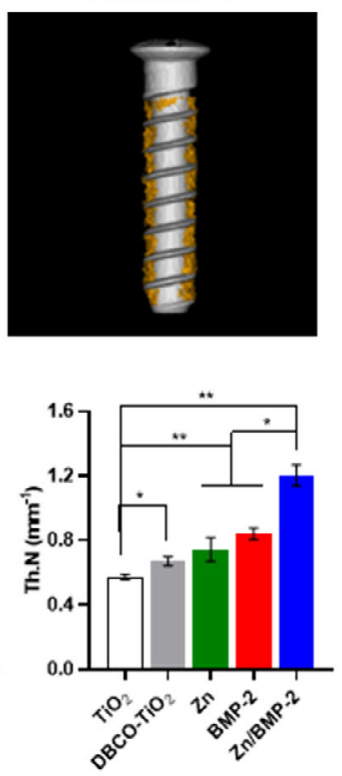

Zn/BMP-2
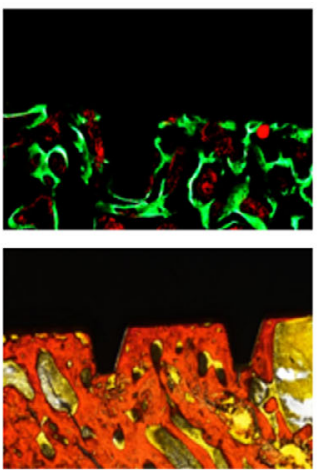

Fig. 7 Micro-CT and histological analysis on osseointegration between bone tissue and screw. A Micro-CT $3 D$ reconstructed images and (B) quantitatively evaluating the peri-implant bone generation according to the BMD, BV/TV, Tb.Sp, Tb.Th and Tb.N (B $n=$ three biologically independent

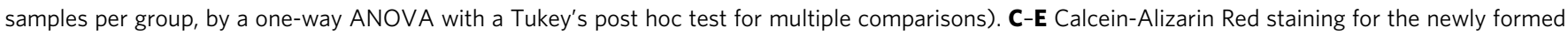
bone and (D) quantitative staining analysis; Van Gieson and bone implant contact (BIC). F Maximum fixation force in different groups determined by pull-

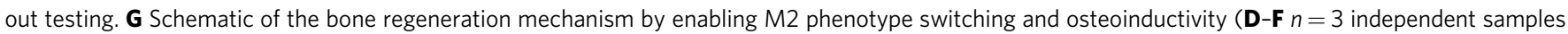

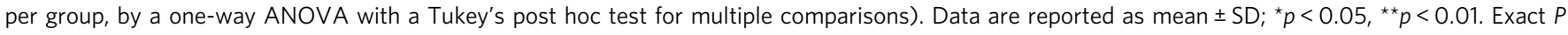
values were given in the Source Data file.

(e.g., $\mathrm{Zn}^{2+}$ ) with bioactive peptide (e.g., BMP-2-derived peptide) to overcome the shortcomings of the traditional methods. It not only ensures the long-term bioactivity of peptide, but also combines unique biological activities of the inorganic metal ions with bioactive peptide to meet the various needs of biological materials. The two-step approach has successfully combined
$\mathrm{Zn}^{2+}$ with BMP-2 derived peptide, acting as a distant modulator for immune cytokine production in the first stage and achieving local activation/differentiation of recruited target cell clusters (e.g., osteogenic cells instead of immune cells) in the second stage. Given that previous works on bone-implant surface modification focus either on osteoinduction or immunomodulation, our work 
is the first to combine the dual functions of molecules and ions for biomodification on bone implants via a mussel adhesionmediated approach. In addition, BMP-2-derived peptide or zinc ion could be replaced by other biomimetic peptides (e.g., VEGF, AMP) and metal ions (e.g., $\mathrm{Cu}^{2+}, \mathrm{Mg}^{2+}$ ) to synthesize varieties of multifunctional coatings for satisfying different clinical requirements. Although further exploration is still needed to understand the potential mechanisms of osteoimmunomodulation, these results have demonstrated a promising strategy toward bone regeneration and bone-implant osseointegration, which is in all probability utilized in future clinical practice and applied to orthopedic research. Furthermore, our mussel adhesion-mediated and molecular bioclickable strategy provides a favorable ossteointegration approach to clinical applications in osteoporosis, diabetes, infection, and poor bone healing. The combination of inorganic metal ions with bioactive peptides and biomaterials will provide more opportunities for developing a new generation of engineering bone implants for orthopedic medicine.

In summary, we here reported a dual-effect coating on bone implants with both immunomodulatory and osteoinductive activities by a mussel adhesion-mediated ion coordination and molecular clicking strategy. The strategy could provide a simple method for co-modification of Ti bone implants with immunoactive metal ions (e.g., $\mathrm{Zn}^{2+}$ ) and osteoinductive growth factors (e.g., BMP-2 peptide). The $\mathrm{Zn}^{2+}$ and BMP-2 peptide comodified implants could elicit a favorable osteoimmune microenvironment by macrophage switch from M1 to M2 phenotypes that facilitates the osteogenic differentiation of BM-MSCs, thus enhancing osseointegration at the bone-implant interface and improving their mechanical stability in vivo. Overall, the dualeffect coating could be utilized to regulate macrophage phenotypic conversion and create a favorable immunomodulatory microenvironment for bone regeneration and osseointegration, providing a new idea of bone tissue engineering implants with immunoactivity and osteoinductivity.

\section{Methods}

Materials. The clickable mussel-derived peptides were prepared using standard Fmoc solid-phase synthesis ${ }^{47}$. Quartz wafers $(10 \mathrm{~mm}$ or $15 \mathrm{~mm}$ in diameter) with $80-100 \mathrm{~nm} \mathrm{TiO}_{2}$ layer were prepared by the key Laboratory of Advanced Technologies of Materials, Southwest Jiaotong University (Chengdu, China). For in vivo experiments, pure Ti screws $(2 \mathrm{~mm} \times 10 \mathrm{~mm})$ were purchased from Tianjin Zhengtian Medical Device Company (Tianjin, China). Cell counting kit-8 (CCK-8) kit, a Live/Dead cell staining kit and FITC-labeled Phalloidin staining were purchased from Yeasen Biotechnology Co. (Shanghai, China). Alkaline phosphatase (ALP) kit, ARS kit and Triton X-100 were obtained from Beyotime Biotechnology Co. (Jiangsu, China). The other chemical reagents or antibodies unless mentioned elsewhere were almost purchased from Sigma, Abcam or Invitrogen.

Surface modification. The peptide coating was performed by immersing the clean $\mathrm{TiO}_{2}$-coated quartz wafers or medical Ti screws in the PBS solution of (DOPA) ${ }_{6}$ $\mathrm{PEG}_{5}-\mathrm{DBCO}(0.01 \mathrm{mg} / \mathrm{mL})$ for $24 \mathrm{~h}$. Note that the peptide solution needs to be first purged with nitrogen $\left(\mathrm{N}_{2}\right)$ for 15 min to reduce the oxidation of catechols. The (DOPA) $)_{6}-\mathrm{PEG}_{5}$-DBCO-coated substrates or screws were then incubated with $0.05 \mathrm{M}$ zinc acetate $\left(\mathrm{Zn}\left(\mathrm{CH}_{3} \mathrm{COO}\right)_{2}, \mathrm{ZnAc}_{2}\right)$ in MiniQ water $(18.2 \mathrm{M} \Omega \mathrm{cm})$ for $12 \mathrm{~h}$. Finally, $\mathrm{Zn}$-loaded substrates or screws were incubated with (2-Azide)-PEG ${ }_{5}$ BMP-2 $(0.1 \mathrm{mg} / \mathrm{mL})$ in PBS solution for $12 \mathrm{~h}$. The $\mathrm{Zn}^{2+}$ and BMP-2 peptide comodified substrates or screws were carefully rinsed with MiniQ water and dried with $\mathrm{N}_{2}$ for further use. All experiments were performed at room temperature.

\section{Characterizations. The two synthetic peptides were first purified by high-} performance liquid chromatography (HPLC, Agilent system with Kromasil 100$5 \mathrm{C} 18$ column) and then evaluated by electrospray ionization mass spectrometry (ESI-MS, Sciex API 150EX LC/MS with Agilent 1100 HPLC). The surface morphology of modified or unmodified $\mathrm{TiO}_{2}$ substrates was evaluated by field emission scanning electron microscope (FE-SEM, Sirion 200, FEI) and atomic force microscopes (AFM, NT-MDT, Russia). The chemical composition of different samples was characterized by energy-dispersive X-ray spectrometry (EDS, Sirion 200, FEI) and X-ray photoelectron spectroscopy (XPS, AXIS Ultra DLD, Japan). Surface wettability of different samples was analyzed by a contact angle instrument Theta Lite (Biolin scientific, Finland). QCM-D (Q-sense AB, Sweden) was used to determine the mass of peptides modified on the $\mathrm{TiO}_{2}$ surfaces. The concentrations

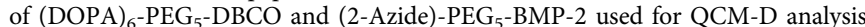
were the same as those used for the peptide coating and bio-orthogonal co-grafting process. To explore the stability of (2-Azide)-PEG 5 -BMP-2 graft on the $\mathrm{TiO}_{2}$ surface, first (2-Azide)-PEG ${ }_{5}$-BMP-2 was labeled with 5-FITC, then the labeled peptides was further used to prepare the $\mathrm{Zn} / \mathrm{BMP}-2$ coating. The fluorescence distribution was observed by a fluorescence microscope (PCOM, Nikon, Japan) after co-culturing Zn/BMP-2 with DMEM for 14 days, and the data was analyzed by ZEN imaging software (Zeiss, Germany). The release behavior of $\mathrm{Zn}^{2+}$ from the Zn/BMP-2 samples in PBS, FBS-free DMEM and 10\% fetal bovine serum (FBS)DMEM was analyzed by an inductively coupled plasma-atomic emission spectrometry (ICP-AES, JY2000-2, France).

Cell culture. RAW264.7 (ATCC, TCM13, Shanghai, China) was provided by the Soochow University (Suzhou, China) as a gift. RAW264.7 cells were cultured in alpha-minimum essential medium ( $\alpha$-MEM, HyClone) supplemented with $10 \%$ FBS and incubated at $37^{\circ} \mathrm{C}$ under $5 \% \mathrm{CO}_{2}$ atmosphere. The cell culture medium of a-MEM was refreshed every two days. Cells $\left(2 \times 10^{4}\right.$ cells/well $)$ were seeded in 24 well plates for the subsequent experiments in vitro.

Bone marrow-derived mesenchymal stem cells (BM-MSCs) were isolated from the 4-week-old male Sprague Dawley (SD) rats (Shanghai Jihui Experimental Animal Center, Shanghai, China) according to a previous protocol ${ }^{70}$. All animal experiments were approved by the Animal Research Committee of Shanghai Jiaotong University School of Medicine. Briefly, the femur and tibia were collected and separated form muscle and connective tissue. After cutting off both ends of the bone, the bone marrow suspensions were flushed out and suspended in Dulbecco's modified Eagle's medium: F-12 (DMEM/F12, HyClone) containing 10\% FBS (Gibco) and $100 \mathrm{U} / \mathrm{ml}$ of penicillin/streptomycin. The cell suspension was filtered by $70 \mu \mathrm{m}$ filters (Millipore, Ireland). The cells were incubated at $37^{\circ} \mathrm{C}$ under $5 \%$ $\mathrm{CO}_{2}$ atmosphere, the medium was refreshed every 2-3 days. When the cells arrived at $80-90 \%$ confluence, the cells were detached from the culture dish by $0.25 \%$ trypsin/EDTA. BM-MSCs were evaluated by flow cytometry in identifying the surface specific markers and confirming their purity before any experiment in vitro. Briefly, passage 3 BM-MSCs were suspended in PBS $(\mathrm{pH}=7.2)$ at a density of $1 \times 10^{6}$ cells/ml and then were stained with FITC anti-rat/mouse CD90 (BioLegend, 202503), PE anti-mouse/rat CD29 (BioLegend, 102207), FITC anti-rat CD45 (BioLegend, 202205) and APC mouse anti-rat CD34 (Novus, NB600-1071) flow cytometry antibodies at $4{ }^{\circ} \mathrm{C}$ for $30 \mathrm{~min}$. Cells without any staining was used as a negative control. Cells were washed in PBS and stained with 7-aminoactinomycin D (7AAD, 559925, Biosciences, BD) according to the manufacture instructions. Quantitative fluorescence analysis was performed with a flow cytometer (LSRFortessa ${ }^{\mathrm{TM}} \mathrm{X}-20, \mathrm{BD}, \mathrm{USA}$ ), and FlowJo ${ }^{\mathrm{TM}}$ software (Version 10.7.1) was used to quantified the expression levels of surface markers.

Cytocompatibility. BM-MSCs and RAW264.7 cells were separately cultured on samples with different surface treatments for $24 \mathrm{~h}$ and gently rinsed with sterilized PBS solution for three times. A Live/Dead cell staining kit (Yeasen, China) consisting of Calcein-AM (green fluorescence) and Propidium iodide (PI, red fluorescence) was used to assess the cell viability. The fluorescent images were acquired by a fluorescence microscope (Nikon, Japan). To further investigate cell viability, cells were collected and incubated with Annexin V-APC and PI. After incubation, binding buffer was added and the cells were analyzed by flow cytometry. All data were analyzed using FlowJo ${ }^{\mathrm{TM}}$ software. Lactate dehydrogenase (LDH, Beyotime, China) and cell counting kit-8 (CCK-8, Yeasen, China) assays were performed to evaluate the cytotoxicity of samples in the cell proliferation. To investigate the adhesion and morphology of BM-MSCs on the samples, FITC-labeled Phalloidin staining (Yeasen, China) and SEM (Sirion 200, FEI) were used in our study. After $24 \mathrm{~h}$ incubation, the samples were fixed with $4 \%$ paraformaldehyde and permeabilized with $0.1 \%$ (v/v) Triton X-100, followed by blocking with $4 \%$ bovine serum albumin (BSA) and staining with FITC-labeled Phalloidin (Yeasen, Shanghai, China) and 4',6-diamidino-2-phenylindole hydrochloride (DAPI, Beyotime, China). Cytoskeletal actin and cell nuclei were observed by a fluorescence microscope (PCOM, Nikon, Japan). Besides fluorescence staining, SEM analysis was also used to investigate the cell adhesion and morphology. After incubating $24 \mathrm{~h}$, cells on different samples surfaces were fixed with glutaraldehyde $(2.5 \% \mathrm{v} / \mathrm{v})$ for $2 \mathrm{~h}$ and dehydrated in gradient ethanol at $30,50,70,85,90$, and $100 \mathrm{v} / \mathrm{v} \%$. Then, the samples were observed by SEM (Sirion 200, FEI).

Macrophage polarization in vitro. In order to investigate the effect of $\mathrm{Zn}^{2+}$ modified surfaces in regulating the polarization of macrophage, $2 \times 10^{4}$ cells/well RAW264.7 cells were cultured on different surfaces in 24 -well plate. Then cells were stimulated with $100 \mathrm{ng} / \mathrm{mL}$ lipopolysaccharide (LPS, Sigma) for $8 \mathrm{~h}$ to induce M1 phenotype. After rinsed in PBS for three times, the complete medium was changed with fresh $\alpha$-MEM medium. After $24 \mathrm{~h}$ culturing, adherent cells were observed by an Olympus CK40 culture microscope (Tokyo, Japan) to investigate the morphology change. The medium supernatants were collected to analyze the secretion of inflammatory cytokines (TNF- $\alpha$ and IL-10) by a commercial mouse cytokine ELISA kits (BD Bioscience, USA). Quantitative real-time polymerase chain reaction (qRT-PCR) was also used to quantify the expression levels of 
inflammatory cytokines (TNF- $\alpha$ and IL-10), surface marker (CCR7, M1 macrophage marker and CD206, M2 macrophage marker), and the osteogenic factors (BMP-2 and VEGF). The total RNA in cells was extracted by Trizol reagent (Invitrogen, USA) according to the manufacturer's instructions. RNA concentrations were quantified by a NanoDrop spectrophotometer (Thermo, USA). Immediately, cDNA was synthesized from $1 \mu \mathrm{g}$ RNA by reverse transcription reaction using a PrimeScript ${ }^{\mathrm{TM}}$ RT reagent Kit (Takara, Japan). Gene expression analysis was performed by a 7500 Real-Time PCR System (Thermo, USA) using SYBR ${ }^{\circledR}$ Premix Ex Taq ${ }^{\mathrm{TM}}$ (Takara, Japan). The sequences of primers were exhibited in the Supporting Information (Supplementary Table 2). In addition, immunofluorescence staining was used to evaluate the polarization of macrophage from M1 to M2 phenotypes. The expression of M1 macrophage-related markers (CD86, iNOS) and M2 macrophage-related markers (CD206, Arg-1) were confirmed by double-staining as previously reported ${ }^{31}$. Briefly, RAW264.7 cells were fixed with $4 \%$ paraformaldehyde (PFA, Sangon Biotech) overnight at $4{ }^{\circ} \mathrm{C}$, permeabilized with $0.2 \%(\mathrm{v} / \mathrm{v})$ Triton X-100 (Sigma, US) for $5 \mathrm{~min}$, blocked with $2 \%$ bovine serum albumin (BSA, Sigma, US) for $1 \mathrm{~h}$ and incubated with primary antibodies at $4{ }^{\circ} \mathrm{C}$ overnight. The primary antibodies included F4/80 (Abcam, ab6640), CD86 (Abclonal, A16805), iNOS (Abcam, ab3523), CD206 (Abcam, ab64693), and Arg-1 (Abcam, ab91279). After incubation, the samples were rinsed with PBS for 5 min and incubated with the goat anti-rabbit IgG H\&L (Alexa Fluor 647, red; Abcam, ab150079) and goat anti-rat IgG H\&L (Alexa Fluor 488, green; Abcam, ab150165) or goat anti-rabbit IgG H\&L (Alexa Fluor 488, green; Abcam, ab150077) antibodies for $1 \mathrm{~h}$ at room temperature. 4,6-diamidino-2-phenylindole (DAPI) was used for nucleus counterstaining and then the samples were imaged by a laser confocal microscopy (LSCM, Zeiss, Germany). Coverslips $(n=3)$ in each group were included for semi-quantitative analysis; and three different subregions were randomly selected. Positive cells and images were analyzed by the Image J (version $1.51 \mathrm{a}, \mathrm{NIH})$ software.

Osteogenic differentiation in vitro. To investigate whether $\mathrm{Zn}^{2+}$-loaded substrates could affect the differentiation of BM-MSCs though modulating the polarization of macrophage, the supernatants of RAW264.7 cells cultured on different surfaces in inflammatory conditions was collected. Then, the supernatant was centrifuged at $\times 60 \mathrm{~g}$ for $5 \mathrm{~min}$ to move any remained cells and frozen at $-80^{\circ} \mathrm{C}$ for further use. In addition, the supernatant filtered with a $0.22 \mu \mathrm{m}$ filter (Millipore, Ireland) was mixed with fresh DMEM/F-12 medium at a ratio of 1:2 to obtain MCM. BM-MSCs was cultured with a density at $2 \times 10^{4}$ cells/well in DMEM/F-12 for $12 \mathrm{~h}$, then the medium was replaced by MCM with osteogenic components ( $10 \mathrm{mM} \beta$-glycerophosphate, $0.1 \mu \mathrm{M}$ dexamethasone and $0.25 \mathrm{mM}$ ascorbate) for further culturing. After culturing for 14 days, ALP staining and activity were performed as previous description ${ }^{31}$. In addition, the ECM mineralization was evaluated by the Alizarin Red Staining $(40 \mathrm{mM}, \mathrm{pH}=4.2$, Cyagen China). Total RNA was extracted from the treated BM-MSCs to measure the amount of osteogenesis-related genes expression (e.g., Alp, Runx2, Colla1, and $O p n)$ by qRT-PCR. The primer sequences for the target genes were listed in Supplementary Table 2. Two osteogenic-related proteins (e.g., ALP, OPN) were evaluated by immunofluorescence staining. Briefly, the cells were fixed in $4 \%$ paraformaldehyde and permeabilized with $0.2 \%$ Triton-X 100, followed by blocking with 2\% BSA and incubating with primary antibodies of ALP (PA5106391, Invitrogen) and OPN (ab63856, Abcam). Subsequently, the cells were respectively incubated with secondary antibody, phalloidin and DAPI. Finally, the cells were observed by a fluorescence microscope (PCOM, Nikon, Japan).

Animal models. All the animal experiments were approved by the Animal Research Committee of Shanghai Jiaotong University School of Medicine, and all the operation procedures were conducted according to National Institutes of Health Guide. Sprague Dawley rats (SD, male, 6-8 weeks old) were randomized into five groups $\left(\mathrm{TiO}_{2}, \mathrm{TiO}_{2}-\mathrm{DBCO}, \mathrm{Zn}, \mathrm{BMP}-2\right.$, and $\left.\mathrm{Zn} / \mathrm{BMP}-2\right)$. The flat lateral surfaces of the femoral condyles were selected as the surgical site. After proper anesthesia and disinfection, the lateral femoral condyle was gradually exposed and was drilled with a $1.5 \mathrm{~mm}$ kirschner wire under the strict asepsis procedure. The Ti screws $(2.0 \mathrm{~mm} \times 10 \mathrm{~mm}$, the height of thread is $0.3 \mathrm{~mm}$, Zhengtian, Tianjing) with $\mathrm{Zn}$ or BMP-2 co-modified surface were placed vertically in bilateral femoral condyles in each animal ( $n=10$ per group). Then, the incision was sutured in separate layers and all the animals was intramuscularly injected with antibiotic for 3 days after surgery.

Macrophage polarization in vivo. Four days after implantation surgery, the animals ( $n=5$ per group) were sacrificed. The bilateral femurs with screws were collected and fixed in $4 \%$ PFA for $48 \mathrm{~h}$, and then decalcified in $10 \%$ ethylenediaminetetraacetic acid (EDTA, Macklin) for following 30 days. After removing the Ti screws, the femurs were embedded in paraffin. Paraffin sections was processed by microtome (Leica, RM2255, Germany) with $5 \mu \mathrm{m}$ thickness for the further staining. H\&E and Goldner's trichrome staining were used to evaluate the inflammation and new collage synthesis around the implants. To evaluate the polarization of macrophages around the implants, the abovementioned sections were stained with CD68 (a pan-macrophages marker; Abcam, ab201340), CCR7 (M1 maker; Servicebio, GB11502), and CD206 (M2 maker; Servicebio, GB13438). In addition, the standard immunohistochemistry was performed to identify IL-10 with primary antibodies against IL-10 (1:100 dilution, Servicebio) according to the manufacturer's instructions. To semi-quantitative analysis, we randomly chose three dependent sections from three different animals in each group to calculate the number of positive cells for CD68, CCR7, CD206, and IL-10 by Image J (version 1.51a, NIH) software.

Micro-CT evaluation. After 8 weeks of implantation, the rats ( $n=5$ per group) were sacrificed. The femurs were isolated and fixed in $70 \%$ alcohol for 3 days. Then, those femurs were scanned by a Micro-CT system (SkyScan1172 Ex-Vivo MicroCT, Belgium) with a rotation step of $0.15^{\circ}$, a pixel size of $17.81 \mu \mathrm{m}, 80 \mathrm{kV}$ source voltage, $112 \mu \mathrm{A}$ source current, $0.5 \mathrm{~mm} \mathrm{Al}$ filter optimizing the contrast, and $370 \mathrm{~ms}$ exposure time. Multilevel thresholding procedure (threshold for new bone $=50-80$, threshold for implant $=130$ ) was applied to distinguish bone from other tissues. The VOI included the trabecular compartment between the outer diameter and inner diameter from the longitudinal axis of the screw. Specifically, the VOI was selected in an axisymmetric cuboid with a circular plane $2 \mathrm{~mm}$ $(d=17.81 \mu \mathrm{m} \times 113$ layer) from the top view (B3 in Supplementary Fig. 12) and a depth of $6 \mathrm{~mm}(\mathrm{~L}=17.81 \mu \mathrm{m} \times 340$ layer $)$ along the longitudinal axis of the screw (B1 and B2 in Supplementary Fig. 12). The 3D images were reconstructed by NRecon software (Version1.7.3.0, Bruker, Kontich, Belgium) with correction for misalignment and ring artefacts. Diversity index of bone regeneration including bone tissue volume/total tissue volume (BV/TV), BMD, trabecular thickness (Tb.Th), trabecular number (Tb.N), and trabecular separation (Tb.Sp) were calculated by supporting analyzing software (CTAn, Bruker, Kontich, Belgium). To further investigate the maximum load of different screws, a biomechanical pull-out testing was performed ${ }^{1}$. The screw was pulled out with a displacement rate of $3 \mathrm{~mm} / \mathrm{min}$ by a material testing system (HY1080, China). The test was stopped when the screw was completely separated from the bone. Meanwhile, the maximum load was recorded and used to evaluate the mechanical stability. Each groups had three parallel replicates.

Histological evaluation. To evaluate bone mineralization in vivo, calcein $(20 \mathrm{mg} /$ $\mathrm{kg}$, Sigma) and alizarin red (30 mg/kg, Sigma) were intraperitoneally injected into the rats, 4 and 6 weeks after the surgery, respectively. After micro-CT examination and fixnation in $70 \%$ alcohol, those femurs around the implantation site were dehydrated in alcohol and acetone. Then these femurs were embedded in methyl methacrylate solution, and processed to get femoral sections with $5 \mu$ m thickness. All the fluorescent-labeled bone sections were visualized by LSCM (Zeiss, Germany). In addition, the sections were stained with van Gieson dye and observed by an optical microscope (Nikon, Japan) in the BIC. Finally, images were acquired and analyzed by Image J software to determine the mineralization rate and BIC.

Statistics and reproducibility. The results were presented as the means \pm SD for each group. The data were analyzed by a one-way ANOVA followed by Tukey's post hoc test for multiple comparisons using the GraphPad Prism software 8.0 (Solvusoft, US). Differences between two groups were considered significant when the $p$ value is $<0.05$. All micrograph assays were carried out at least three independent times with similar results.

Reporting summary. Further information on research design is available in the Nature Research Reporting Summary linked to this article.

\section{Data availability}

The authors declare that all data supporting the findings of this study are available within this paper and its Supplementary Information. All data is available from the corresponding authors upon reasonable request. The Source data underlying Figs. 2C, D, F, H, J-L, N, O, 3D, E, 4D-F, H, I, K, L, M-R, 5E-L, 6D-H, and 7B, D-F; Supplementary Figs. 2, 3C, D, 4, 6B, 7, 9B, C, 10B, 11B and 15 are provided as a Source data file. Source data are provided with this paper.

Received: 10 April 2021; Accepted: 7 December 2021; Published online: 10 January 2022

\section{References}

1. Pan, G. et al. Biomimetic design of mussel-derived bioactive peptides for dualfunctionalization of titanium-based biomaterials. J. Am. Chem. Soc. 138, 15078-15086 (2016)

2. Wang, T. et al. Synergistic effects of titania nanotubes and silicon to enhance the osteogenic activity. Colloids Surf. B Biointerfac. 171, 419-426 (2018).

3. Tejero, R., Anitua, E. \& Orive, G. Toward the biomimetic implant surface: Biopolymers on titanium-based implants for bone regeneration. Prog. Polym. Sci. 39, 1406-1447 (2014). 
4. Bayliss, L. E. et al. The effect of patient age at intervention on risk of implant revision after total replacement of the hip or knee: a population-based cohort study. Lancet 389, 1424-1430 (2017).

5. Ferguson, R. J. et al. Hip replacement. Lancet 392, 1662-1671 (2018).

6. Wu, X., Chen, S., Ji, W. \& Shi, B. The risk factors of early implant failure: a retrospective study of 6113 implants. Clin. Implant Dent. Relat. Res 23, 280-288 (2021).

7. Niu, Y., Wang, Z., Shi, Y., Dong, L. \& Wang, C. Modulating macrophage activities to promote endogenous bone regeneration: Biological mechanisms and engineering approaches. Bioact. Mater. 6, 244-261 (2021).

8. Ellis, S., Lin, E. J. \& Tartar, D. Immunology of wound healing. Curr. Dermatol Rep. 7, 350-358 (2018)

9. Koons, G. L., Diba, M. \& Mikos, A. G. Materials design for bone-tissue engineering. Nat. Rev. Mater. 5, 584-603 (2020).

10. Zhu, Y. et al. Modulation of macrophages by bioactive glass/sodium alginate hydrogel is crucial in skin regeneration enhancement. Biomaterials 256, $120216(2020)$

11. Takizawa, T. et al. Titanium fiber plates for bone tissue repair. Adv. Mater. 30, 1703608 (2018).

12. Sridharan, R., Cameron, A. R., Kelly, D. J., Kearney, C. J. \& O’Brien, F. J. Biomaterial based modulation of macrophage polarization: a review and suggested design principles. Mater. Today 18, 313-325 (2015).

13. Li, C. M. et al. Design of biodegradable, implantable devices towards clinical translation. Nat. Rev. Mater. 5, 61-81 (2020).

14. Xie, Y. J. et al. Osteoimmunomodulatory effects of biomaterial modification strategies on macrophage polarization and bone regeneration. Regen. Biomater. 7, 233-245 (2020).

15. Takayanagi, H. Osteoimmunology in 2014: Two-faced immunology-from osteogenesis to bone resorption. Nat. Rev. Rheumatol. 11, 74-76 (2015).

16. Oishi, Y. \& Manabe, I. Macrophages in inflammation, repair and regeneration. Int Immunol. 30, 511-528 (2018).

17. Zhu, Y. et al. Regulation of macrophage polarization through surface topography design to facilitate implant-to-bone osteointegration. Sci. Adv. 7, eabf6654 (2021).

18. Visalakshan, R. M. et al. Biomaterial surface hydrophobicity-mediated serum protein adsorption and immune responses. ACS Appl. Mater. Interfac. 11, 27615-27623 (2019).

19. Chen, L. et al. Nanostructural surfaces with different elastic moduli regulate the immune response by stretching macrophages. Nano Lett. 19, 3480-3489 (2019).

20. Lv, L. et al. Unveiling the mechanism of surface hydrophilicity-modulated macrophage polarization. Adv. Health. Mater. 7, e1800675 (2018).

21. Li, J., Jiang, X., Li, H., Gelinsky, M. \& Gu, Z. Tailoring materials for modulation of macrophage fate. Adv. Mater. 33, e2004172 (2021).

22. Jin, S. S. et al. A biomimetic hierarchical nanointerface orchestrates macrophage polarization and mesenchymal stem cell recruitment to promote endogenous bone regeneration. ACS Nano 13, 6581-6595 (2019).

23. Vasconcelos, D. P. et al. Modulation of the inflammatory response to chitosan through M2 macrophage polarization using pro-resolution mediators. Biomaterials 37, 116-123 (2015).

24. $\mathrm{Hu}, \mathrm{Z}$., Ma, C., Rong, X., Zou, S. \& Liu, X. Immunomodulatory ECM-like microspheres for accelerated bone regeneration in diabetes mellitus. ACS Appl. Mater. Interfac. 10, 2377-2390 (2018).

25. Vieira, S. et al. Self-mineralizing Ca-enriched methacrylated gellan gum beads for bone tissue engineering. Acta Biomater. 93, 74-85 (2019).

26. Lee, C. H., Kim, Y. J., Jang, J. H. \& Park, J. W. Modulating macrophage polarization with divalent cations in nanostructured titanium implant surfaces. Nanotechnology 27, 085101 (2016).

27. Zhao, D. W. et al. Strontium-zinc phosphate chemical conversion coating improves the osseointegration of titanium implants by regulating macrophage polarization. Chem. Eng. J. 408, 127362 (2021).

28. Liu, W. et al. Zinc-modified sulfonated polyetheretherketone surface with immunomodulatory function for guiding cell fate and bone regeneration. Adv. Sci. 5, 1800749 (2018)

29. Maitz, M. F. et al. The blood compatibility challenge. Part 4: Surface modification for hemocompatible materials: Passive and active approaches to guide blood-material interactions. Acta Biomater. 94, 33-43 (2019)

30. Chen, X., Sevilla, P. \& Aparicio, C. Surface biofunctionalization by covalent co-immobilization of oligopeptides. Coll. Surf. B Biointerfac. 107, 189-197 (2013).

31. Bai, J. et al. Biomimetic osteogenic peptide with mussel adhesion and osteoimmunomodulatory functions to ameliorate interfacial osseointegration under chronic inflammation. Biomaterials 255, 120197 (2020).

32. Hou, Y., Deng, X. \& Xie, C. Biomaterial surface modification for underwater adhesion. Smart Mater. Med. 1, 77-91 (2020).

33. Zhao, X. et al. Silicon-doped titanium dioxide nanotubes promoted bone formation on titanium implants. Int. J. Mol. Sci. 17, 292 (2016).
34. Cychy, S., Hiltrop, D., Andronescu, C., Muhler, M. \& Schuhmann, W Operando thin-layer atr-ftir spectroelectrochemical radial flow cell with tilt correction and borehole electrode. Anal. Chem. 91, 14323-14331 (2019).

35. Qin, W. et al. Osseointegration and biosafety of graphene oxide wrapped porous CF/PEEK composites as implantable materials: The role of surface structure and chemistry. Dent. Mater. 36, 1289-1302 (2020).

36. Guo, S. et al. A vessel subtype beneficial for osteogenesis enhanced by strontium-doped sodium titanate nanorods by modulating macrophage polarization. J. Mater. Chem. B 8, 6048-6058 (2020).

37. Ren, X. et al. Surface modification and endothelialization of biomaterials as potential scaffolds for vascular tissue engineering applications. Chem. Soc. Rev. 44, 5680-5742 (2015)

38. $\mathrm{Yu}, \mathrm{H}$. et al. Nitric oxide-generating compound and bio-clickable peptide mimic for synergistically tailoring surface anti-thrombogenic and antimicrobial dual-functions. Bioact. Mater. 6, 1618-1627 (2021).

39. Chen, X., Gao, Y., Wang, Y. \& Pan, G. Mussel-inspired peptide mimicking: an emerging strategy for surface bioengineering of medical implants. Smart Mater. Med. 2, 26-37 (2021).

40. Lee, H., Lee, B. P. \& Messersmith, P. B. A reversible wet/dry adhesive inspired by mussels and geckos. Nature 448, 338-341 (2007).

41. Zhang, X., Chen, G., Yu, Y., Sun, L. \& Zhao, Y. Bioinspired adhesive and antibacterial microneedles for versatile transdermal drug delivery. Res.-China 2020, 3672120 (2020).

42. Ryu, J. H., Messersmith, P. B. \& Lee, H. Polydopamine surface chemistry: a decade of discovery. Acs Appl Mater. Inter. 10, 7523-7540 (2018).

43. Yang, Y. et al. Endothelium-mimicking multifunctional coating modified cardiovascular stents via a stepwise metal-catechol-(amine) surface engineering strategy. Res.-China 2020, 9203906 (2020).

44. Li, Y. et al. Molecular design principles of Lysine-DOPA wet adhesion. Nat. Commun. 11, 3895 (2020)

45. Akram Bhuiyan, M. S. et al. In situ deactivation of catechol-containing adhesive using electrochemistry. J. Am. Chem. Soc. 142, 4631-4638 (2020)

46. Ding, Y. H., Floren, M. \& Tan, W. Mussel-inspired polydopamine for biosurface functionalization. Biosurf. Biotribol. 2, 121-136 (2016).

47. Yang, Z. et al. Bioclickable and mussel adhesive peptide mimics for engineering vascular stent surfaces. Proc. Natl Acad. Sci. USA 117, 16127-16137 (2020).

48. Czuban, M. et al. Titanium coating with mussel inspired polymer and bioorthogonal chemistry enhances antimicrobial activity against Staphylococcus aureus. Mater. Sci. Eng. C. 116, 111109 (2020).

49. Gai, M. et al. A bio-orthogonal functionalization strategy for site-specific coupling of antibodies on vesicle surfaces after self-assembly. Polym. Chem. 11, 527-540 (2020).

50. Sasmal, R. et al. Synthetic host-guest assembly in cells and tissues: Fast, stable and selective bioorthogonal imaging via molecular recognition. Anal. Chem. 90, 11305-11314 (2018).

51. Ahmad, N. et al. Peptide cross-linked poly(2-oxazoline) as a sensor material for the detection of proteases with a quartz crystal microbalance. Biomacromolecules 20, 2506-2514 (2019).

52. Shi, Z., Neoh, K. G., Kang, E. T., Poh, C. \& Wang, W. Titanium with surfacegrafted dextran and immobilized bone morphogenetic protein-2 for inhibition of bacterial adhesion and enhancement of osteoblast functions. Tissue Eng. Part A 15, 417-426 (2009).

53. Cho, H.-j, et al. Effective immobilization of bmp-2 mediated by polydopamine coating on biodegradable nanofibers for enhanced in vivo bone formation. Acs Appl. Mater. Inter 6, 11225-11235 (2014).

54. Xiang, G. et al. In situ regulation of macrophage polarization to enhance osseointegration under diabetic conditions using injectable silk/sitagliptin gel scaffolds. Adv. Sci. 8, 2002328 (2021).

55. Wang, Z. et al. Switching on and off macrophages by a "bridge-burning" coating improves bone-implant integration under osteoporosis. Adv. Funct. Mater. 31, 2007408 (2020).

56. Rao, A. J. et al. Revision joint replacement, wear particles, and macrophage polarization. Acta Biomater. 8, 2815-2823 (2012).

57. Armiento, A. R., Hatt, L. P., Sanchez Rosenberg, G., Thompson, K. \& Stoddart, M. J. Functional biomaterials for bone regeneration: a lesson in complex biology. Adv. Funct. Mater. 30, 1909874 (2020).

58. Bonaventura, P., Benedetti, G., Albarède, F. \& Miossec, P. Zinc and its role in immunity and inflammation. Autoimmun. Rev. 14, 277-285 (2015).

59. Maares, M. \& Haase, H. Zinc and immunity: an essential interrelation. Arch. Biochem Biophys. 611, 58-65 (2016).

60. Chen, Z. et al. Osteoimmunomodulation for the development of advanced bone biomaterials. Mater. Today 19, 304-321 (2016).

61. McWhorter, F. Y., Wang, T., Nguyen, P., Chung, T. \& Liu, W. F. Modulation of macrophage phenotype by cell shape. Proc. Natl Acad. Sci. USA 110, 17253-17258 (2013).

62. Kang, H. et al. Immunoregulation of macrophages by dynamic ligand presentation via ligand-cation coordination. Nat. Commun. 10, 1696 (2019). 
63. Sadtler, K. et al. Design, clinical translation and immunological response of biomaterials in regenerative medicine. Nat. Rev. Mater. 1, 16040 (2016).

64. Shu, Y. et al. The immunomodulatory role of sulfated chitosan in BMP-2mediated bone regeneration. Biomater. Sci. 6, 2496-2507 (2018).

65. Holloway, W. R., Collier, F. M., Herbst, R. E., Hodge, J. M. \& Nicholson, G. C. Osteoblast-mediated effects of zinc on isolated rat osteoclasts: Inhibition of bone resorption and enhancement of osteoclast number. Bone 19, 137-142 (1996).

66. Li, Y. et al. Enhanced osseointegration and antibacterial action of zinc-loaded titania-nanotube-coated titanium substrates: In vitro and in vivo studies. $J$. Biomed. Mater. Res A 102, 3939-3950 (2014).

67. Rahman, C. V. et al. Controlled release of BMP-2 from a sintered polymer scaffold enhances bone repair in a mouse calvarial defect model. J. Tissue Eng. Regen. Med. 8, 59-66 (2014).

68. Shao, N. et al. Development of organic/inorganic compatible and sustainably bioactive composites for effective bone regeneration. Biomacromolecules 19, 3637-3648 (2018).

69. Zhou, X. et al. BMP-2 derived peptide and dexamethasone incorporated mesoporous silica nanoparticles for enhanced osteogenic differentiation of bone mesenchymal stem cells. Acs Appl. Mater. Inter. 7, 15777-15789 (2015).

70. Zhu, H. et al. A protocol for isolation and culture of mesenchymal stem cells from mouse compact bone. Nat. Protoc. 5, 550-560 (2010).

\section{Acknowledgements}

This work was supported by the National Natural Science Foundation of China (81972134 and 82102535), National Key Research and Development Program of China (2018YFC1106200), Shanghai Municipal Education Commission-Gaofeng Clinical Medicine Grant Support (20171906), Shanghai Jiao Tong University "Medical and Research" Program (YG2021ZD06 and ZH2018ZDA04), China Postdoctoral Science Foundation Funded Project (2019M661560), and GuangCi Professorship Program of Ruijin Hospital Shanghai Jiao Tong University School of Medicine.

\section{Author contributions}

T.W. and J.B. performed materials characterization, cell culture, in vitro experiment. L.W. and G.C. guided the in vivo experiments. T.W., C.H., and M.L. performed in vivo implantation. D.G. and J.Q. provided assistant in Micro-CT evaluation and histology analysis. W.C. and T.W. designed and synthesized the peptides. W.C. and L.D. conceived, directed and supervised the study. W.C. and L.D. analyzed the data and wrote the paper with help from all authors. All authors discussed the results and commented on the paper.

\section{Competing interests}

The authors declare no competing interests.

\section{Additional information}

Supplementary information The online version contains supplementary material available at https://doi.org/10.1038/s41467-021-27816-1.

Correspondence and requests for materials should be addressed to Jin Qi, Wenguo Cui or Lianfu Deng.

Peer review information Nature Communications thanks Michael Pujari-Palmer and the other anonymous reviewers for their contribution to the peer review of this work. Peer reviewer reports are available.

Reprints and permission information is available at http://www.nature.com/reprints

Publisher's note Springer Nature remains neutral with regard to jurisdictional claims in published maps and institutional affiliations.

cc (i) Open Access This article is licensed under a Creative Commons Attribution 4.0 International License, which permits use, sharing, adaptation, distribution and reproduction in any medium or format, as long as you give appropriate credit to the original author(s) and the source, provide a link to the Creative Commons license, and indicate if changes were made. The images or other third party material in this article are included in the article's Creative Commons license, unless indicated otherwise in a credit line to the material. If material is not included in the article's Creative Commons license and your intended use is not permitted by statutory regulation or exceeds the permitted use, you will need to obtain permission directly from the copyright holder. To view a copy of this license, visit http://creativecommons.org/ licenses/by/4.0/

(C) The Author(s) 2022 